\title{
Comparative assessment of pressure field reconstructions from particle image velocimetry measurements and Lagrangian particle tracking
}

\author{
P. L. van Gent ${ }^{1}$ D. Michaelis ${ }^{2}$ B. W. van Oudheusden ${ }^{1}$ P. -É. Weiss ${ }^{3} \cdot$ R. de Kat ${ }^{4}$ - A. Laskari ${ }^{4}$ - Y. J. Jeon ${ }^{5}$

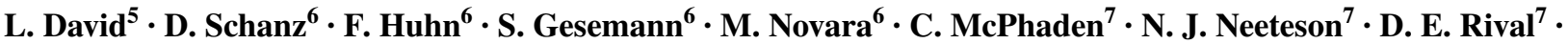 \\ J. F. G. Schneiders ${ }^{1}$ F. F. J. Schrijer ${ }^{1}$
}

Received: 29 August 2016 / Revised: 27 February 2017 / Accepted: 28 February 2017 / Published online: 27 March 2017

(C) The Author(s) 2017. This article is an open access publication

\begin{abstract}
A test case for pressure field reconstruction from particle image velocimetry (PIV) and Lagrangian particle tracking (LPT) has been developed by constructing a simulated experiment from a zonal detached eddy simulation for an axisymmetric base flow at Mach 0.7. The test case comprises sequences of four subsequent particle images (representing multi-pulse data) as well as continuous time-resolved data which can realistically only be obtained for low-speed flows. Particle images were processed using tomographic PIV processing as well as the LPT algorithm 'Shake-The-Box' (STB). Multiple pressure field reconstruction techniques have subsequently been applied to the PIV results (Eulerian approach, iterative least-square pseudo-tracking, Taylor's hypothesis approach, and instantaneous Vortex-in-Cell) and LPT results (FlowFit, Vortex-in-Cell-plus, Voronoi-based pressure evaluation, and iterative least-square pseudo-tracking).
\end{abstract}

P. L. van Gent

p.l.vangent@tudelft.nl

1 Faculty of Aerospace Engineering, Delft University of Technology, Delft, The Netherlands

2 LaVision GmbH, Göttingen, Germany

3 ONERA, The French Aerospace Lab, F-92190 Meudon, France

4 Engineering and the Environment, University of Southhampton, Southampton, UK

5 Institut PPRIME, UPR3346, CNRS-Université de PoitiersISAE-ENSMA, Poitiers, France

6 Department of Experimental Methods, German Aerospace Center (DLR), Institute of Aerodynamics and Flow Technology, Göttingen, Germany

7 Department of Mechanical and Materials Engineering, Queen's University, Kingston, Canada
All methods were able to reconstruct the main features of the instantaneous pressure fields, including methods that reconstruct pressure from a single PIV velocity snapshot. Highly accurate reconstructed pressure fields could be obtained using LPT approaches in combination with more advanced techniques. In general, the use of longer series of time-resolved input data, when available, allows more accurate pressure field reconstruction. Noise in the input data typically reduces the accuracy of the reconstructed pressure fields, but none of the techniques proved to be critically sensitive to the amount of noise added in the present test case.

\section{Introduction}

Fluid pressure is directly related to phenomena like surface loading and sound generation (aeroacoustics) and as such is an important quantity in many engineering problems. While surface pressure can experimentally be determined with pressure transducers and pressure-sensitive paint (PSP), pressure field reconstruction based on particle image velocimetry (PIV) and Lagrangian particle tracking (LPT) offers a number of unique advantages (van Oudheusden 2013). The term LPT instead of PTV is used here to signify that Lagrangian information about position, velocity, and acceleration of individual particles is used in our study. Contrary to the more established measurement techniques, PIV/LPT-based pressure field reconstruction does not require instrumentation or surface preparation of the wind tunnel model. This allows for pressure determination in configurations where such modifications are not practical, e.g., very thin (or membrane-like) airfoils. In more regular configurations, it avoids the installation of large numbers of pressure transducers which is a common practice to 
obtain reliable surface load distributions. Another unique feature of PIV/LPT-based pressure field reconstruction is its inherent ability to provide simultaneous velocity and pressure data in the full flow field, thus enabling a better insight in the relation between fluid dynamics and surface loads or sound fields.

Given these beneficial features, there has been a long interest in PIV/LPT-based pressure field reconstruction. Many studies have addressed different implementations of the technique (van Oudheusden 2013). In recent years, the technique has become increasingly feasible and appealing, especially due to the development of (time-resolved) volumetric diagnostic capabilities, such as tomographic PIV (Elsinga et al. 2006; Scarano 2013) and Lagrangian particle tracking (Schanz et al. 2016). With the maturing of the method, its scale-up from small-size research environments is currently receiving attention in collaborative European framework programs like 'AFDAR' and 'NIOPLEX'. The particular work described in the current paper reflects a joint effort by partners in 'NIOPLEX' together with DLR Göttingen, and to the authors' knowledge constitutes the first comparative study, in which multiple research groups work on the same test case (similar as in the "PIV challenges").

\section{Overview of PIV/LPT-based pressure field reconstruction techniques}

In PIV/LPT-based pressure field reconstruction, kinematic data obtained with PIV/LPT are used to compute the local pressure gradient $(\nabla p)$ using the momentum equation (Eq. 1). Pressure is subsequently obtained through spatial integration:

$\nabla p=-\rho \frac{\mathrm{Du}}{\mathrm{D} t}+\mu \nabla^{2} \mathbf{u}$,

where $\rho$ is the density, $\mu$ is the kinematic viscosity, and $\mathrm{Du} / \mathrm{D} t$ is the material acceleration, which is the acceleration of a fluid parcel in a Lagrangian perspective. The viscous term (right) can be neglected for sufficiently high Reynolds numbers. For incompressible flow, the density is a constant. Under compressible flow conditions, it can be eliminated as independent variable by combining the momentum and energy equations (van Oudheusden et al. 2007) (see Sect. 6.1). Note that by time-averaging of Eq. 1, mean pressure fields can be obtained from uncorrelated velocity fields (Gurka et al. 1999).

The experimental determination of the material acceleration has been subject of extensive research. Different methods have been proposed. Using two or more PIV velocity fields closely separated in time, the material acceleration can be determined via a pseudo-Lagrangian formulation by tracing imaginary fluid particles using a series of velocity fields (e.g., Liu and Katz 2006), also referred to as pseudo-tracking. Alternatively, the material acceleration can be estimated via an Eulerian formulation by separately determining local temporal and spatial velocity derivatives from the structured velocity measurement grid (e.g., Baur and Köngeter 1999). To alleviate the need for timeresolved velocity data, methods have been proposed that employ physical models to calculate the material acceleration from velocity data at a single time instance, e.g., Taylor's hypothesis approach (de Kat and Ganapathisubramani 2013; Laskari et al. 2016) and Instantaneous Vortex-in-Cell (IVIC, Schneiders et al. 2016).

Alternative to taking velocity fields as a starting point, the material acceleration can also be obtained from particle images, by tracking particle patterns or individual particles. The first approach is based on correlation analyses and includes fluid trajectory correlation (FTC, Lynch and Scarano 2013) or fluid trajectory evaluation based on an ensemble-averaged cross-correlation (FTEE, Jeon et al. 2014). The latter strategy includes the conventional particle tracking velocimetry (PTV) that relies on relatively low particle seeding concentrations (e.g., Malik et al. 1993) as well as two more recently proposed advanced approaches that avoid this drawback: tomographic-PTV resp. LPT (Schröder et al. 2011; Novara and Scarano 2013) and 'Shake-The-Box' (STB, Schanz et al. 2016). Different techniques exist to reconstruct scattered LPT measurement data on a uniform grid, e.g., FlowFit (Gesemann et al. 2016) and Vortex-in-Cell+ (Schneiders and Scarano 2016).

In addition, for spatial integration of the pressure gradient (Eq. 1), different procedures exist: two main methods are direct numerical integration along various paths in space (e.g., Baur and Köngeter 1999; Liu and Katz 2006) and formulation and solving of a Poisson equation (e.g., de Kat and van Oudheusden 2012). The two approaches are compared in Charonko et al. (2010). Recently, a number of novel techniques has been proposed and tested: Tronchin et al. (2015) sequentially integrate different subdomains to limit spatial error propagation. Jeon et al. (2015) minimise the difference between measured and reconstructed pressure gradients in a least-square sense (Least-square method). Huhn et al. (2016) employ the fast Fourier transform (FFT integration). The implementations of the techniques mentioned above all require input data on a structured grid. To allow for a direct use of scattered measurement data from particle tracking, two recently proposed techniques perform spatial integration of scattered data: Gesemann et al. (2016) define the pressure field using 3D cubic B-splines, the parameters of which are obtained from minimizing a cost function (FlowFit2). Neeteson and Rival (2015) apply a finite-volume discretisation based on Voronoi tessellation. 
The performance of PIV/LPT-based pressure field reconstruction is influenced by the characteristics of the flow as well as the quality of the measurement data. The relative strengths, weaknesses, and dependencies of different approaches in this respect have been addressed in a number of comparative performance assessments [see van Oudheusden (2013) for a review]. The present study contributes to these efforts using a wide variety of state-of-theart velocimetry-based pressure determination techniques (see Table 1) to obtain pressure from the same synthetic particle-image data. The techniques are classified in PIVbased techniques, which rely on the velocity data obtained with cross-correlation of particle images/volumes, and LPT-based techniques, which require tracking of individual particles. A further distinction is made between techniques which only require velocity data at a single time instance only (3 and 4) and techniques that require time-resolved input data (all others). All LPT processings for the present study are performed using the 'Shake-The-Box' algorithm.

\section{Description of the test case}

The test case consists of the flow over an axisymmetric step, which forms a simplified representation of the flow over the base of a launcher during its ascent phase. The flow over the launcher base gives rise to fluctuating side loads on the nozzle. Quantifying these loads is critical for the efficient design of nozzles and surrounding structures. Apart from this relevance to industry, the choice for the test case is motivated by its richness in terms of spatial and temporal flow scales and its challenging nature that is inherent to high-speed, three-dimensional, separatingreattaching flows. It should be remarked here that not all applied techniques perform optimal for the flow conditions under consideration and this test case alone, therefore, does not constitute an exhaustive performance assessment of the different methods. These limitations should be kept in mind when interpreting the results of the study.

To have reference data available against which the pressure results can be validated, the test case is based on a numerical simulation. A PIV/LPT experiment is simulated by creating synthetic particle images of a virtual measurement volume. Model geometry (see Fig. 1) and flow conditions are designed to represent typical experimental arrangements in the $27 \times 27 \mathrm{~cm}^{2}$ transonic-supersonic wind tunnel at the Aerodynamics Laboratory at Delft University of Technology.

The model main body has a diameter $(D)$ of $50 \mathrm{~mm}$. The afterbody has a diameter $(d)$ of $20 \mathrm{~mm}(0.4 D)$, resulting in a step height of $15 \mathrm{~mm}(0.3 \mathrm{D})$. Wind tunnel walls are omitted in the simulation for simplicity and robustness. The free stream flow has a Mach number $\left(\mathrm{M}_{\infty}\right)$ of 0.7 , a total pressure $\left(p_{t}\right)$ of $200 \mathrm{kPa}$, and a total temperature $\left(T_{t}\right)$ of $285 \mathrm{~K}$.

Table 1 Overview of pressure evaluation techniques considered in this study (see Sect. 6 for more detailed descriptions)

\begin{tabular}{lllll}
\hline No. & Abbreviations & Velocity measurement & Determination of material acceleration & Integration of pressure gradient \\
\hline $1 \mathrm{a}$ & ILAG & PIV & Pseudo-tracking & Least-square method \\
$1 \mathrm{~b}$ & & PIV (FTEE) & & Poisson solver \\
$1 \mathrm{c}$ & & LPT & Eulerian approach & Poisson solver \\
2 & EUL & PIV & Taylor's hypothesis approach & Poisson solver \\
3 & TH & PIV $(1$ snapshot $)$ & Instantaneous Vortex-in-Cell & FFT integration \\
4 & IVIC & PIV $(1$ snapshot $)$ & & FlowFit B \\
5 & FFA & LPT + FlowFit A & & Poisson solver \\
6 & FFB & LPT & & Voronoi-based \\
7 & VIC + & LPT + Vortex-in-Cell-plus & LPT & \\
8 & VOR & & & \\
\hline
\end{tabular}

Fig. 1 Sketch of the geometry; diameter $D$ equals $50 \mathrm{~mm}$. Filled coloured contours depict the mean streamwise velocity in the virtual measurement volume for the simulated experiment

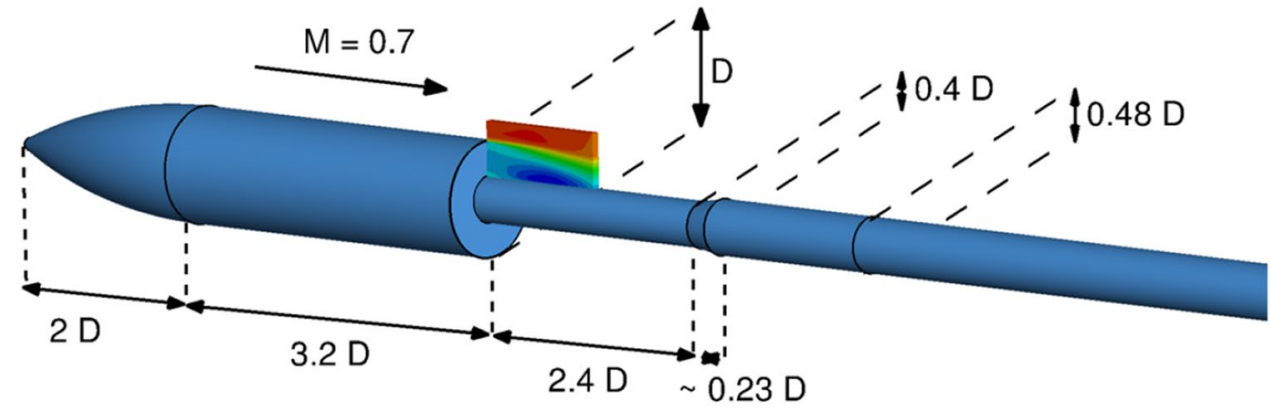


Using the isentropic flow relations, this corresponds to a free stream pressure of $144,186 \mathrm{~Pa}$, a free stream temperature of $260 \mathrm{~K}$, and a free stream velocity $\left(\mathrm{U}_{\infty}\right)$ of $226 \mathrm{~m} \mathrm{~s}^{-1}$. The Reynolds number based on the model main body diameter $\left(\operatorname{Re}_{\mathrm{D}}\right)$ is 1.3 million. The measurement domain of the simulated experiment has a size of $1.2 D \times 0.47 D \times 0.08 D$ (60 mm $\times 24 \mathrm{~mm} \times 4 \mathrm{~mm}, L \times H \times W$ ) (see Fig. 3).

Synthetic particle images are processed using tomographic PIV algorithms (Elsinga et al. 2006) as well as with the LPT algorithm 'Shake-The-Box' (STB, Schanz et al. 2016). This enables the use of PIV-based pressure determination techniques that require velocity input data on a Cartesian grid, as well as LPT-based techniques which take scattered data available at particle positions as starting point. To be able to assess the impact of measurement noise, processing is performed using both idealized, noise-free particle images (clean) as well as particle images to which a controlled amount of artificial noise is added (noisy).

The test case comprises continuous time-resolved data as well as sequences of four subsequent images, from here on referred to as multi-pulse data. This distinction is motived by considerations with respect to the limitations encountered under actual measurement conditions. The multi-pulse data can actually be made available for highspeed flows by using PIV systems with multiple independent laser and camera systems (e.g., Souverein et al. 2009; Schröder et al. 2013; Lynch and Scarano 2014a). Continuous series of time-resolved data on the other hand cannot realistically be obtained for the present flow case (i.e., a high-speed, subsonic, compressible flow) within the limits of current measurement capabilities. For low-speed flows, modern high-speed laser and camera equipment do, however, allow sufficiently high sample rates to perform timeresolved measurements. It is, therefore, deemed insightful to consider the time-resolved data sets as a means to assess pressure extraction capabilities for (low-speed) flow conditions.

Figure 2 shows the organisation of the particle images and velocity fields with respect to time. The smaller time separation of $2 \mu \mathrm{s}$ is equal to the time separation for PIV processing. The larger time separation of $10 \mu$ s used in the multi-pulse data was found to be suitable as time separation for determining the material acceleration based on a preliminary assessment by Blinde et al. (2014).

Table 2 provides an overview of the data sets that comprise the test case. The data sets have been distributed to different research groups as a blind test case. To re-create the situation after an experiment, reference data from the numerical simulation were not distributed and kept isolated from the researchers that applied the pressure field reconstruction techniques. For the same reason, different time instances have been considered for the 'clean' and 'noisy' cases, i.e., cases without or with artificial noise added to the particle images. If the time instances had been the same, then the researchers could have easily determined the exact noise levels by comparing the two cases. To facilitate the sharing of data, only a limited number of time instances were considered.

\section{Details of simulated experiment}

\subsection{Zonal detached eddy simulation (ZDES)}

A dedicated ZDES simulation (Deck 2005, 2012) is performed building on ample experience with applying ZDES to axisymmetric base flows (e.g., Deck and Thorigny 2007; Weiss et al. 2009; Weiss and Deck 2011). ZDES is closely related to the classical detached eddy simulation (DES). The main difference between the two approaches is that within ZDES, the user has to select the Reynolds-averaged
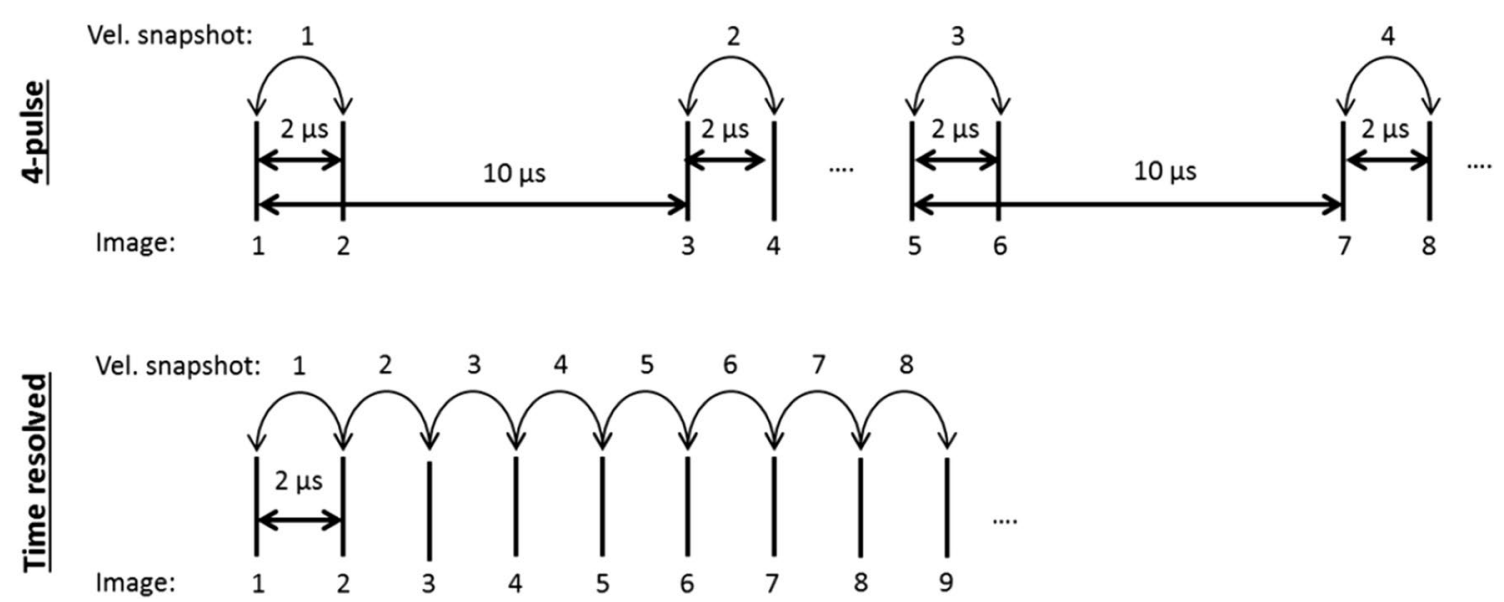

Fig. 2 Time-separation diagrams for consecutive particle images, 4-pulse data (top), and continuous time-resolved data (bottom) 
Table 2 Overview of data sets that comprise the test case. 'Clean' and 'Noisy' refer to the cases without or with artificial noise added to the particle images

\begin{tabular}{ll}
\hline Type & Description \\
\hline $\begin{array}{l}\text { Continuous time-resolved data } \\
\text { ('clean' and 'noisy') }\end{array}$ & $\begin{array}{l}\text { Particles images at } 42 \text { consecutive time instances } \\
41 \text { velocity snapshots obtained through PIV processing } \\
\text { Velocity and acceleration data obtained through LPT processing at the same } 41 \text { time instance as PIV velocity } \\
\text { fields }\end{array}$ \\
& 21 sets of particle images at 4 consecutive time instances \\
& 21 sets of 2 consecutive velocity snapshots obtained through PIV processing \\
4-pulse data ('clean' and 'noisy' & Velocity and acceleration data obtained through LPT processing at the middle time instances of the sets of \\
& particle images/velocities \\
& Mean and r.m.s. velocity fields based on 5000 snapshots obtained through PIV processing \\
Calibration images for four virtual cameras & Velocity, density, and pressure data from the numerical simulation interpolated to the PIV grid positions \\
Camera calibration & Velocity, density, and pressure data from the numerical simulation interpolated to the particle positions as \\
Reference PIV & determined by LPT \\
Reference LPT &
\end{tabular}

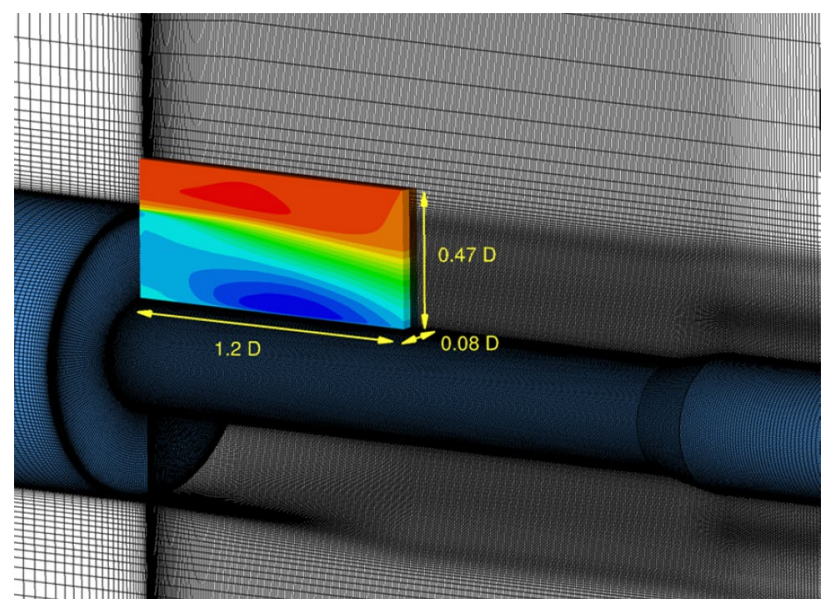

Fig. 3 Detail of mesh with sketch of the geometry; filled coloured contours depict the mean streamwise velocity in the virtual measurement volume for the simulated experiment

Navier-Stokes (RANS) and DES domains. The selection of the appropriate mode depends on the nature of the flow problem. Given that the flow separation is fixed by the geometry, a mode I (i.e., DES) has been retained for the separated area and a mode 0 (i.e. URANS) for the region upstream from the separation occurring at the trailing edge of the main body.

Model geometry and flow conditions have been presented in Sect. 3. The simulation uses a cylindrical grid with 17 million points of which 10 million points are located in the separated area. The virtual PIV/LPT measurement volume contains close to 300,000 points (see Fig. 3). The grid is locally refined at the location of the shear layer. Calculations are performed with a time-step $\left(\Delta \mathrm{t}_{\text {ZDES }}\right)$ of $0.5 \mu \mathrm{s} .5000$ snapshots are sampled with a time separation $2 \mu \mathrm{s}\left(\Delta \mathrm{t}_{\text {sampling }}\right)$, covering a total duration of $10 \mathrm{~ms}$. The temporal resolution was designed to meet the time separation of the PIV recording. According to the Nyquist-Shannon criterion, this sampling allows to resolve temporal scales with Strouhal numbers $\left(\mathrm{St}_{\mathrm{D}}\right)$ from 0.02 to 55 . The output data used for this study consist of velocity, density, and pressure values.

Figure 4 shows the mean streamwise velocity, mean pressure, as well as the turbulence intensity and the root mean square (r.m.s) of the pressure fluctuations in a radial plane of the simulated domain. Here, the pressure coefficient $\left(\mathrm{C}_{\mathrm{p}}\right)$ and the turbulence intensity (T.I.) are defined as follows:

$\mathrm{C}_{\mathrm{p}}=\frac{p-p_{\infty}}{q_{\infty}}=\frac{\frac{p}{p_{\infty}}-1}{\frac{1}{2} \gamma M_{\infty}^{2}}$,

T.I. $=\sqrt{\frac{1}{3}\left(\left(u_{\mathrm{RMS}}^{\prime}\right)^{2}+\left(v_{\mathrm{RMS}}^{\prime}\right)^{2}+\left(w_{\mathrm{RMS}}^{\prime}\right)^{2}\right)} / U_{\infty}$.

In these equations, $q_{\infty}$ denotes the freestream dynamic pressure, $\gamma$ denotes the ratio of specific heats, and $u_{\mathrm{RMS}}^{\prime}$, $v_{\mathrm{RMS}}^{\prime}$, and $w_{\mathrm{RMS}}^{\prime}$ denote the r.m.s. of the fluctuating velocity components in $x$-, $y$-, and $z$-directions, respectively. Results have been obtained on the basis 5000 time samples and considering all planes in azimuthal direction to improve the statistical convergence. The bottom-left figure shows the location of the measurement volume for the simulated experiment (see dashed box).

The mean flow field (top-left figure) shows a shear layer that emanates from the corner of the step at $y / D=0.3$ and grows in downstream direction. The mean reattachment of the shear layer occurs at approximately $x / D=1.25$, which is just outside the virtual PIV measurement volume. Below the shear layer, a recirculation region occurs, where the mean velocity reaches a minimum of $-0.30 \mathrm{U}_{\infty}$, while the minimum mean pressure $\left(\mathrm{C}_{\mathrm{p}, \min }\right)$ is -0.23 . A high pressure region is present 

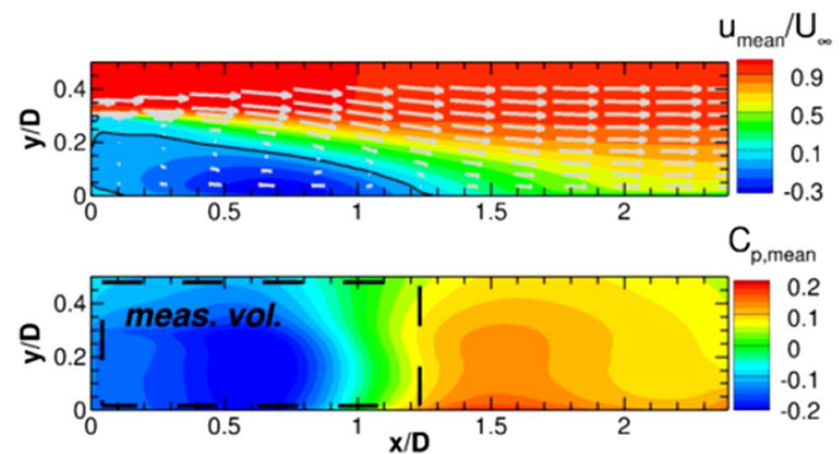

Fig. 4 Mean streamwise velocity (top-left), mean pressure (bottomleft), turbulence intensity (top-right), and r.m.s. of normalised pressure fluctuations (bottom-right); top-left figure: black solid line indi-

downstream of the point of mean reattachment, with a maximum mean pressure $\left(\mathrm{C}_{\mathrm{p}, \max }\right)$ of 0.18 at $x / D=1.50$. The figures on the right show elevated levels of turbulence intensity and pressure fluctuations in the shear layer and the reattachment region, with maxima of T.I. $\max =0.30$ and $\mathrm{C}_{p \text { RMS, } \max }^{\prime}=0.08$ located at about $x / D=1.0 ; y / D=0.2$.

Figure 5 depicts a representative realisation of the instantaneous streamwise velocity, density, pressure, and streamwise material acceleration. Especially, the material acceleration field (bottom-right) shows small-scale flow structures originating from the corner of the step that break down towards the reattachment region which is characterised by interactions of flow structures with a variety of length scales.

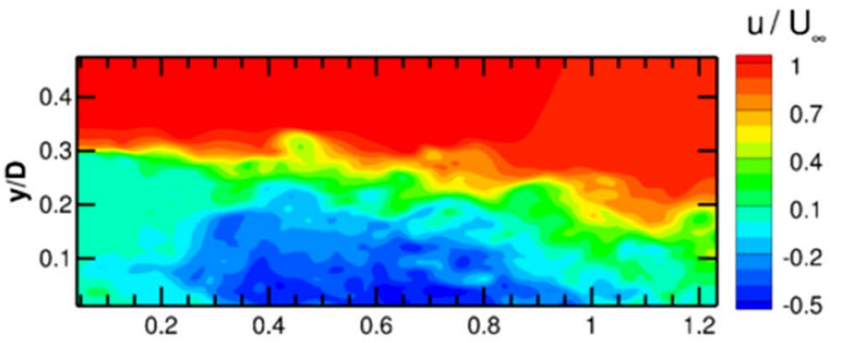

$\mathrm{C}_{\mathrm{0}}$

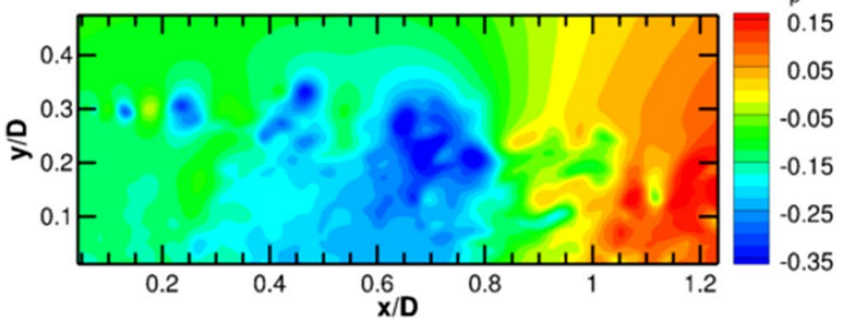

T.I.

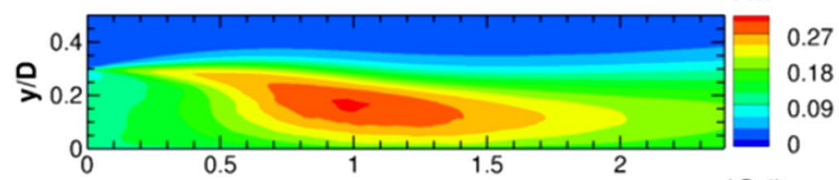

$\left(\mathrm{C}_{\mathrm{p}}^{\prime}\right)_{\mathrm{RMS}}$

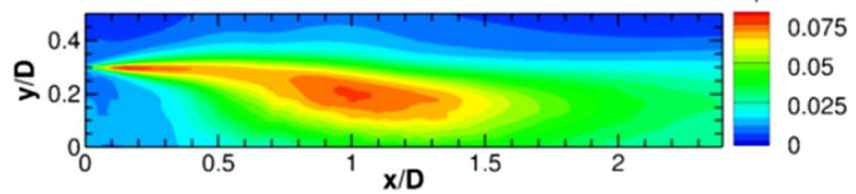

cates zero mean streamwise velocity; vectors have been subsampled for clarity; bottom-left figure: dashed box indicates the measurement volume of the simulated experiment

\subsection{Calculation of particle tracks}

The measurement domain is randomly seeded with particles, so that the particle images contain 0.05 particles per pixel (ppp). The particles are propagated using an explicit, fourth-order Runge-Kutta method in combination with spline interpolation in time and natural neighbour interpolation in space. Three time integration steps are performed per simulation sampling time-step of $2 \mu \mathrm{s}$, so that the Courant number is smaller than 1 and the Courant-Friedrichs-Lewy (CFL) condition is met in the full domain with the exception of a thin region in the direct vicinity of the corner of the step. The measurement volume is surrounded by a buffer region. After each time-step, particles in this region are removed after which it is randomly seeded with new particles. The region is large enough, so that there are always particles available to
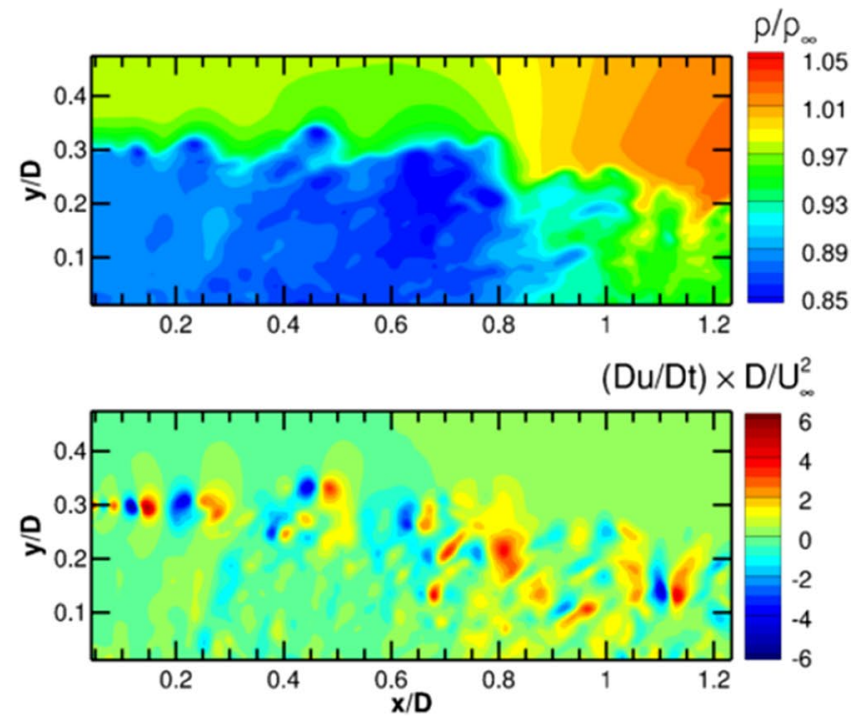

Fig. 5 Instantaneous velocity (top-left), density (top-right), pressure (bottom-left), and streamwise material acceleration (bottom-right) 
flow into the domain and the seeding concentration remains constant.

The instantaneous particle velocity is taken equal to the instantaneous fluid velocity at the particle position, which is a valid approach for the great majority of the flow field, where the flow timescales are smaller than the typical seeding particle relaxation time $\left(\tau_{\mathrm{p}}\right)$ of approximately $2 \mu$ s (Ragni et al. 2010). To assess the numerical errors from integration and interpolation, particles are integrated one sample timestep forward and subsequently backward in time. Comparing the resulting position with the original position yields a local error estimate of below $10^{-5} \mathrm{U}_{\infty},(<0.0001$ voxel displacement). In a second test, targeted at quantifying the errors due to interpolation, particle positions are integrated one sample time-step forward in time using the original simulation data and the simulation data subsampled at half the spatial and temporal resolution. Comparing the resulting positions and assuming the error scales with a factor $2^{-4}$ of the spatial and temporal resolution (valid for fourth-order methods) yield typical local r.m.s. error estimates of $<0.003 \mathrm{U}_{\infty}(<0.03$ voxel displacement).

\subsection{Generation of synthetic particle images}

The settings used for generating the synthetic images are chosen to represent typical PIV measurements of highspeed flows. Particle images are recorded every $2 \mu$ s by four virtual cameras with a chip size of $1624 \times 800$ pixels and a pixel pitch of $4.4 \mu \mathrm{m}$. The three-dimensional particle positions are projected onto the two-dimensional sensors by perspective projection (Hartley and Zimmermann 2003). First, the position of points in the measurement volume is defined with respect to the coordinate system defined by the camera (Eq. 4). Next, the transformed positions are projected onto the image plane using a pinhole camera model (Eq. 5):

$\mathbf{x}_{\text {cam }}=\mathbf{R}\left(\mathbf{x}_{\text {meas.vol }}-\mathbf{T}\right)$,

$\mathbf{x}_{\text {img }}=\left[\begin{array}{l}x_{\text {img }} \\ y_{\text {img }}\end{array}\right]=\frac{f}{z_{\text {cam }}}\left[\begin{array}{l}x_{\text {cam }} \\ y_{\text {cam }}\end{array}\right]$, where $\mathbf{x}_{\text {meas.vol }}, \mathbf{x}_{\mathbf{c a m}}$, and $\mathbf{x}_{\mathbf{i m g}}$ denote positions in the coordinate systems of the measurement volume, of the camera, and of the image, respectively. $\mathbf{R}$ and $\mathbf{T}$ are the rotation matrix and translation vector of the camera transform (see Hartley and Zimmermann 2003), respectively, and $f$ is the axial distance from the camera centre to the image plane.

The four cameras are placed in a cross-configuration with yaw and pitch angles of $\pm 30^{\circ}$ to achieve a total system aperture of $60^{\circ}$, an optimal configuration for tomographic reconstruction (Scarano 2013). The cameras are equipped with lenses with a focal length of $75 \mathrm{~mm}$ and placed at a virtual distance of $0.83 \mathrm{~m}$ resulting in a magnification of 0.12 and a digital resolution of 22.9 voxel $\mathrm{mm}^{-1}$. The resulting particle displacement in the free stream is about 11 voxels.

Particle images are obtained using a similar approach as outlined in Lecordier and Westerweel (2004). The intensity of projected particles is modelled to follow a Gaussian distribution; a valid approximation for particles of which the geometric projection is smaller than their diffraction spot size. Particle images are obtained using 2D Gaussian integration. The intensity of each pixel is taken as superposition of all contributions of particles. This speckle-free approximation is valid for source densities $\left(\mathrm{N}_{\mathrm{s}}\right)$ below the speckle limit of $\mathrm{N}_{\mathrm{s}}=0.30$.

Two sets of images are generated: a set of idealized particle images (Fig. 6, left) and another set of noisy particle images (Fig. 6, right). The idealized images contain particles with a peak intensity of 512 counts and a diffraction spot size of 2 pixels, resulting in a source density $\left(\mathrm{N}_{\mathrm{s}}\right)$ of 0.16 . The intensity of the background is zero. The noisy particle images contain particles with a nominal peak intensity of 342 counts and a diffraction spot size of 2.5 pixels, resulting in a source density $\left(\mathrm{N}_{\mathrm{s}}\right)$ of 0.25 . For the noisy case, the seeding is modelled to consist of $\mathrm{TiO}_{2}$ particles with a primary crystal size of $55 \mathrm{~nm}$ which are known to form agglomerates with a mean size of about $400 \mathrm{~nm}$ (Schrijer et al. 2006). Here, we randomly generate particle diameters from a Gaussian distribution with mean of $400 \mathrm{~nm}$ and a standard deviation of $100 \mathrm{~nm}$. The distribution is truncated at two standard deviations, to eliminate
Fig. 6 Extracts of a particle image; clean (left) and noisy (right). In this example, the extracts depict the same region at the same time instance
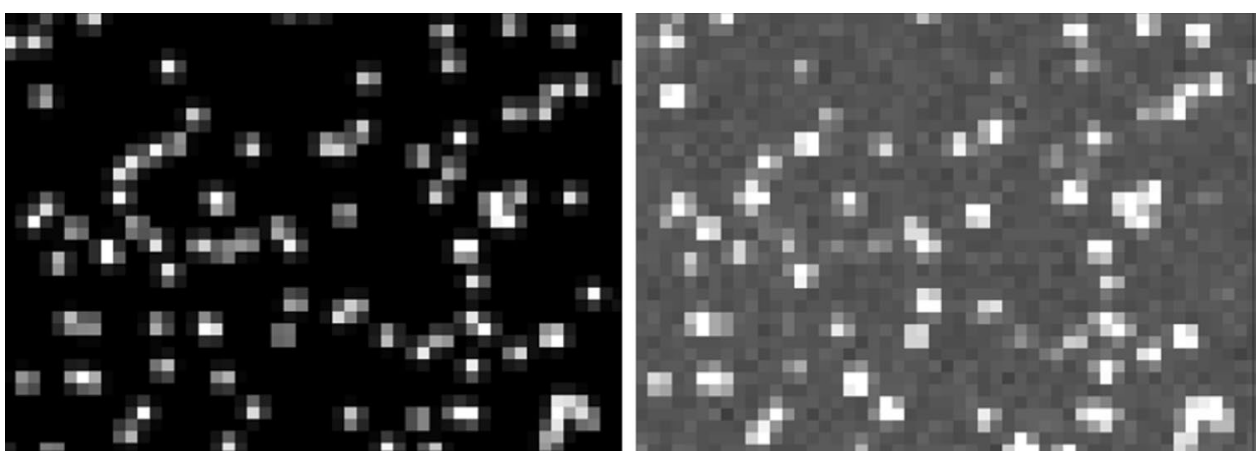
extremely small and large particles. The peak intensity is modelled to vary with the 4th-power of the physical particle diameter (Adrian and Yao 1985), resulting in particle peak intensities range from 21 to 1731 counts. The particle diameter and peak intensity is attributed to a particle once and does not change through time or per camera. Two noise sources are added: shot noise and thermal noise. Shot noise is dependent on the pixel intensity and is implemented, so that for every pixel, the intensity is a random realisation from a Poisson distribution with mean that is equal to the intensity of the noise-free image. Thermal noise is independent of the intensity and is generated as random realisations from a Poisson distribution with a mean value of 170 counts. Mean thermal noise is subtracted before further processing.

Despite efforts to make the synthetic experimental data representative of realistic experiments, they remain of unrealistically high quality in the sense that they benefits from an optimal seeding distribution, optimal camera viewing angles, uniform illumination intensity, an aberration-free optical projection model, a CCD fill ratio of one, absence of any unwanted light reflections, and non-uniform refraction due to density variations of the flow. The time-resolved data and multi-pulse data both have been given similar characteristics in the present study. In reality, however, time-resolved measurements typically have different characteristics, in particular a lower image quality due to the relatively small light budget of high-speed lasers and the relatively large pixel size of high-speed cameras.

\subsection{Tomographic PIV processing}

Particle images have been processed using tomographic PIV algorithms available in the LaVision 8.2 software. The FTEE algorithm was applied using Institut PPRIME in-house codes. The virtual cameras are calibrated in a two-step approach that mimics the typical procedure in an experiment. In step one, a geometric calibration is obtained using images of a virtual calibration plate that is designed to mimic images of typical geometric calibration plates used in experiments. More specifically, the plate consists of a black background with a grid-like pattern of 3-pixel wide points with a spacing of $0.1 \mathrm{D}$. The virtual calibration plate is placed in three different positions (at the centre of the thin volume as well as at its edges of the volume). In step two, volume self-calibration (Wieneke 2008) is performed using actual particle images.

Reconstructed volumes are obtained using seven iterations of the fast MART algorithm after initialisation with a uniform value of 1.0. A $3 \times 3 \times 3$ Gaussian smoothing is applied after each iteration, excluding the final iteration. The computational efficiency is increased by not updating voxels with intensities below 0.005 counts (Atkinson and
Soria 2009). Based on the intensity distribution in z-direction, the procedure was found to result in signal-to-noise ratios (SNR) of about $>100$ and 12 for the clean and noisy cases, respectively.

Cross-correlation is performed using iterative multi-grid volume deformation (VODIM based on Scarano and Riethmuller 2000), symmetric block direct correlation (Discetti and Astarita 2012), and Gaussian window weighting (Discetti et al. 2013). Vector fields from intermediate correlation steps are enhanced for the next iteration by removing spurious vectors, identified by universal outlier detection (Westerweel and Scarano 2005), replacing them using linear interpolation and by Gaussian smoothing of the velocity field. The final three iterations are performed with an interrogation window size of 32 voxels at $75 \%$ overlap, resulting in a vector spacing of $0.35 \mathrm{~mm}$ ( 8 voxels) and a measurement grid of $171 \times 67 \times 11$ vectors. Assuming a round window with a diameter of 32 voxels, each final (round) window contains about 7 particles. No post-processing was applied to the result of the final iteration.

Cross-correlation analysis is performed using 2 consecutive particle volumes as well as using 9 consecutive volumes with fluid trajectory evaluation based on an ensemble-averaged cross-correlation (FTEE, Jeon et al. 2014). Here, the FTEE algorithm was implemented to fit a secondorder polynomial over 7 consecutive snapshots.

\subsection{LPT processing ('Shake-The-Box')}

For the LPT processing, particle paths are determined using the 'Shake-The-Box' algorithm (STB, Schanz et al. 2016). An adapted version of the STB strategy (Novara et al. 2016a, b) has been applied to the multi-pulse data. All cases were processed using DLR in-house codes.

For the initiation phase of STB, the particle distribution in the first four images is determined using Iterative Particle Reconstruction (IPR, Wieneke 2013). Four normal triangulation iterations are performed $(m=4)$, followed by two triangulation iterations $(n=2)$ using a reduced set of three cameras. Seven so-called shake iterations are executed after each triangulation $(k=7)$. The allowed triangulation error is 0.7 pixel in the clean case and 1.1 pixel in the noisy case. The intensity threshold for particle identification is set to 100 counts and 40 counts, respectively. Tracks are searched in the resulting particle distributions for the four time-steps, using a predictor field obtained by particle space correlation (PSC, Novara et al. 2016b) evaluation. For the timeresolved cases, from the fifth time-step on this predictor is completely replaced by predictions based on already identified particle tracks and the number of triangulation iterations is reduced ( $m=3, n=2, k=7$ ). Three passes of STB are conducted going forwards and backwards in time to extend any non-identified parts of already detected tracks 
and to find any previously overlooked tracks. For each reference particle, a search area having $1 \mathrm{px}$ radius is located at the peak position; if at least one particle tracked by STB is found within the search area, the particle is considered as detected. In the end, the algorithm finds over $99 \%$ of all available particle tracks for the clean case and around 96\% for the noisy case. A third-order B-spline function is fit through the resulting particle tracks to reduce noise and to obtain Lagrangian velocity and acceleration as derivatives of a continuous function. The cut-off frequency of the spline fit is determined using the spectral distribution of the unfitted tracks, like a Wiener filter used for particle position prediction (Gesemann et al. 2016).

Concerning the multi-pulse data, an iterative STB approach strategy is applied (Novara et al. 2016a), where the sequential application of IPR $(m=4, n=2, k=5)$ and tracking allows to progressively increase the number of reconstructed tracks. The allowed triangulation error is 0.6 pixel and 1.1 pixel for the clean and noisy cases, respectively. The intensity threshold for particle identification is set to 20 counts for the clean case, while an adaptive threshold is adopted for the noisy case where the threshold value is progressively reduced for each of the STB iterations. Three STB iterations are applied for the clean case and four for the noisy case. Approximately, 99\% and 88\% of the real four-pulse particle tracks are successfully identified by STB for the clean and noisy cases, respectively. As for the time-resolved case, a third-order B-spline function is fit through the resulting particle tracks. A more detailed description of the processing method is provided by Novara et al. (2016b); an experimental investigation of a transonic jet in air at Mach 0.9 by means of a multi-pulse acquisition system and STB has been presented by Manovski et al. (2016).

\section{Velocity error assessment}

\subsection{PIV velocity error}

Error estimates are obtained by assessing the difference between PIV velocity fields and ZDES simulation data interpolated to the PIV grid points. The mean difference represents the bias error and the standard deviation of the difference represents the random error. Error quantities are expressed in voxels displacement, where 1 voxel displacement corresponds to $0.1 \mathrm{U}_{\infty}$. Bias errors were found to be smaller than 0.1 voxel throughout the measurement volume with the exception of a small thin region at the location of the shear layer in the direct vicinity of the step.

Figure 7 shows the r.m.s. errors of the velocity fields obtained with the clean (left) and noisy (right) particle images. The depicted errors relate to single-pair cross-correlation. The smallest errors occur in the freestream, where the typical error is $<0.1$ voxel, and in the recirculation region. In most of the domain, the error is $<0.5$ voxel. The largest errors can be found in the shear layer and the reattachment region. The error quantities found are within the typical range of uncertainties of tomographic PIV reported for similar flows (Lynch and Scarano 2014a, b; Blinde et al. 2015). Comparison of the clean and noisy cases shows that the typical impact of noise is about 0.05 voxel. The use of the multi-frame PIV processing algorithm FTEE was found to reduce overall error levels by 10 and $20 \%$ for the clean and noisy cases, respectively (see overview in Sect. 5.3).

\subsection{STB velocity error}

The STB velocity error is calculated as the difference between the STB velocity values at the particle positions and the ZDES simulation data interpolated to the particle position and instance. Error statistics are subsequently obtained for spatial bins with a similar size as the PIV vector spacing. Figure 8 shows the r.m.s error on basis of 5000 time-resolved STB results. Whereas STB works with physical units and does not involve the concept of a voxel, the STB velocity error has been rescaled to be expressed in terms of voxel displacement to facilitate comparison with PIV results.

Errors in velocity values obtained from the clean particle images (left figures) are of a similar magnitude as the error estimate for the particle integration procedure, suggesting an almost perfect reconstruction. For all other cases, the error typically stays below 0.05 voxel. Larger errors occur at the in- and outflow where not all tracks are immediately identified and the particle tracks can benefit less from the information from adjacent time instances. Similar features as for the PIV results can be observed, but error levels are an order of a magnitude smaller (note the different scale). Errors for the multi-pulse STB approach are typically 0-0.01 voxel higher (see overview in Sect. 5.3).

\subsection{Comparison of velocity errors}

To further characterise and compare the performance of difference methods, Table 3 provides an overview of relevant error values. Columns for 'Freestream' and 'Reattachment region' represent typical r.m.s. error values in those regions. Column 'Global' provides the r.m.s. error value for the full measurement domain. FTEE results consist of only 29 snapshots per case and no complete error assessment could be carried out due to lack of statistical convergence. Global velocity error for FTEE results has been obtained by first determining the relative improvement with respect to standard cross-correlation for the snapshots considered 


\section{r.m.s. error for clean images}
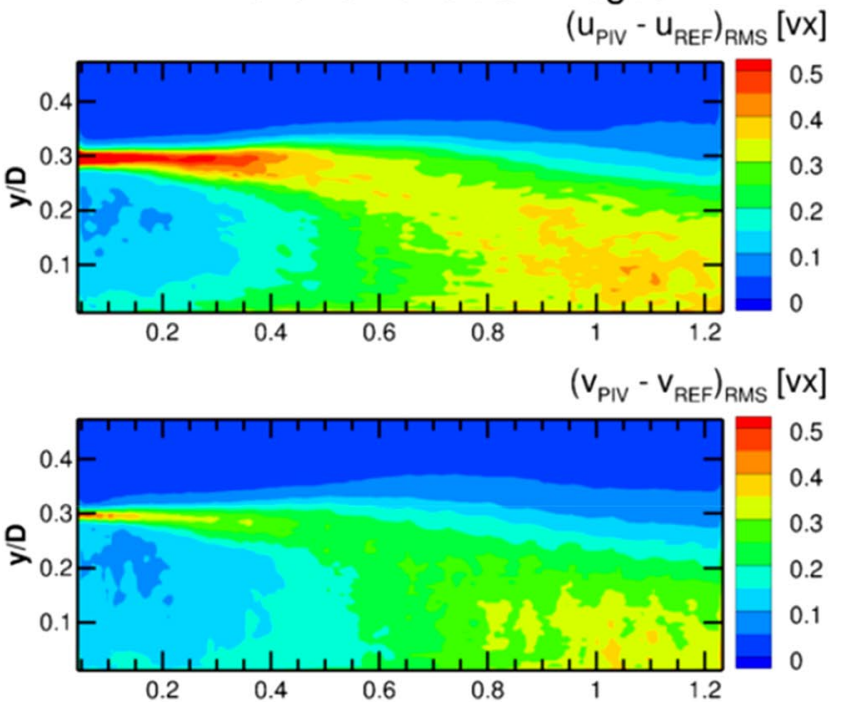

$\left(\mathrm{w}_{\mathrm{PIV}}-\mathrm{w}_{\mathrm{REF}}\right)_{\mathrm{RMS}}[\mathrm{vx}]$

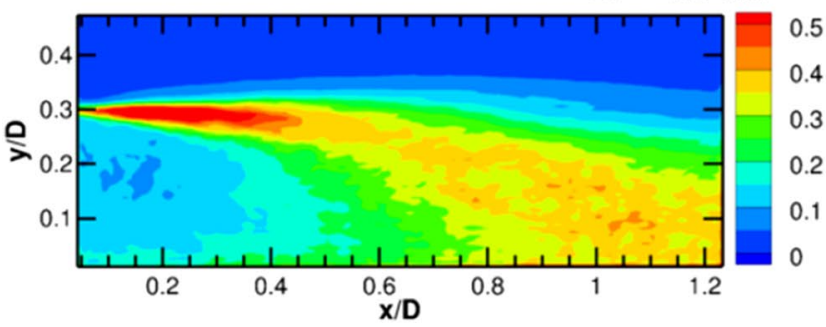

r.m.s. error for noisy images
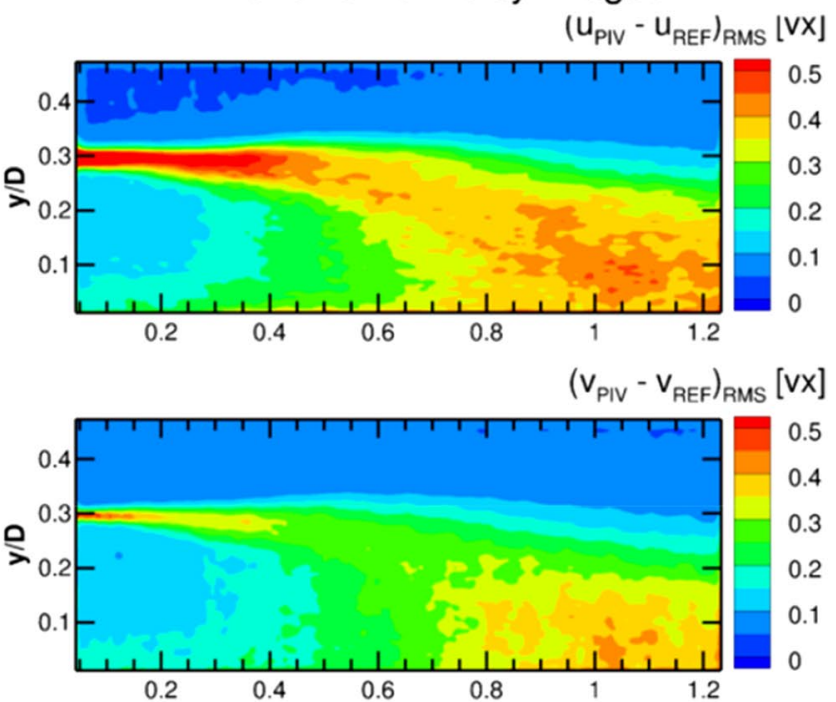

$\left(\mathrm{w}_{\mathrm{PIV}}-\mathrm{w}_{\mathrm{REF}}\right)_{\mathrm{RMS}}[\mathrm{vx}]$

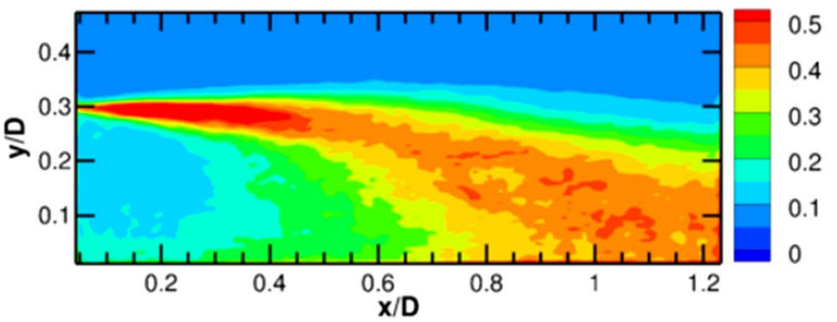

Fig. 7 Centre-plane r.m.s error of velocity components, expressed in voxels displacement obtained by single-pair PIV processing of the clean (left) and noisy (right) particle images; streamwise (top), wall-normal (middle), and out-of-plane (bottom) direction

and subsequently scaling the global velocity error resulting from standard cross-correlation.

The table shows large differences in the observed error levels for PIV and STB, e.g., for the noisy case, there is almost a factor 10 difference in the reattachment region. The lower error levels of STB compared to PIV are consistent with Schanz et al. (2016), who attributed the lower errors from STB to a combination of the absence of ghost particles in the three-dimensional reconstruction, improved particle position accuracy in STB with respect to the position error of the correlation peak in PIV, lack of spatial filtering due to windowing in the PIV analysis, and better temporal coherence.

For the present experiment, the impact of ghost particles can be considered small compared to that of the other error sources. This is evidenced by the high signal-to-noise ratios of the tomographic reconstructions resulting from a combination of optimal camera viewing angles and relatively high image quality. Only marginal improvements could, therefore, be achieved with the advanced tomographic reconstruction methods sequential motion-tracking enhanced MART ((S)MTE, Novara et al. 2010; Lynch and Scarano
2015). The impact of the random error in the measured particle displacement can be estimated from the improvements achieved by the FTEE algorithm which allows reducing the contribution of the random error by considering multiple snapshots. The improvement is more pronounced for the noisy case, since higher noise in the particle images leads to higher random position errors in the locations of the correlation peak.

Finally, Fig. 9 quantifies the impact of spatial filtering by showing the difference between the streamwise velocity from the simulation data interpolated to PIV grid points and a spatially filtered velocity field. Whereas the spatial filtering associated with PIV depends on the specific implementation of the PIV processing as well as the properties of the velocity field (see Schrijer and Scarano 2008; Theunissen 2012), here, it was approximated by a moving average filter; a widely accepted simplification. The filter is implemented by taking the average velocity of all particles (interpolated from the simulation data) within a specified radial distance from the PIV grid points. In taking the average, a Gaussian weighting function was used, implemented similarly as 
r.m.s. error for clean images

$\left(u_{\text {STB }}-u_{\text {REF }}\right)_{\text {RMS }}[v x]$

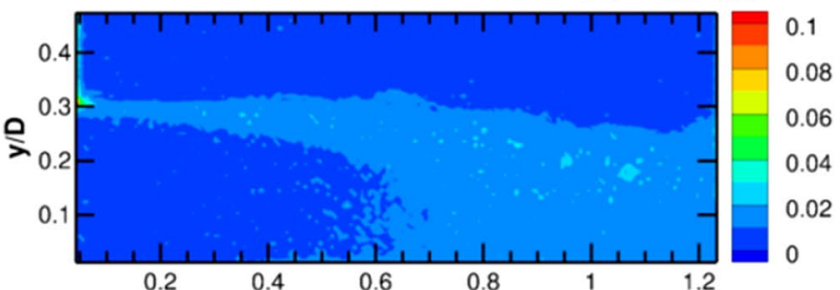

$\left(\mathrm{v}_{\mathrm{STB}}-\mathrm{v}_{\mathrm{REF}}\right)_{\mathrm{RMS}}[\mathrm{vx}]$

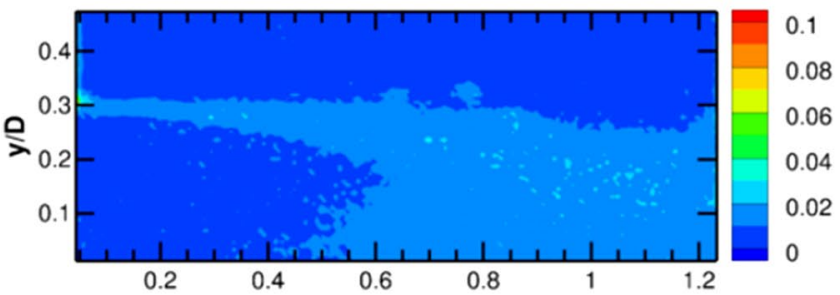

$\left(\mathrm{w}_{\mathrm{STB}}-\mathrm{w}_{\mathrm{REF}}\right)_{\mathrm{RMS}}[\mathrm{vx}]$

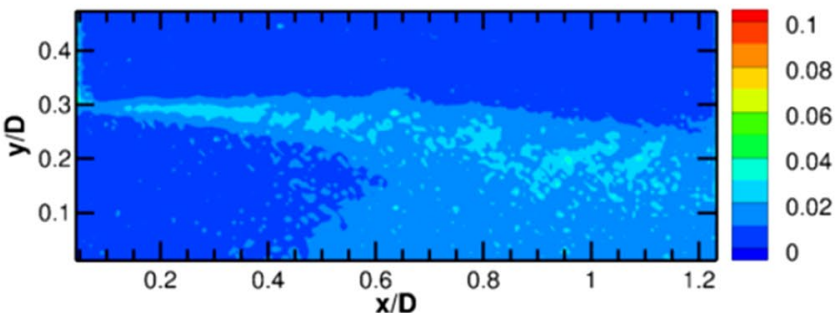

Fig. 8 Centre-plane r.m.s. error of velocity components, expressed in voxels displacement obtained by STB processing of the clean (right) and noisy (left) particle images; streamwise (top), wall-normal (mid-

\section{r.m.s. error for noisy images}

$\left(u_{\text {STB }}-u_{\text {REF }}\right)_{\text {RMS }}[v X]$
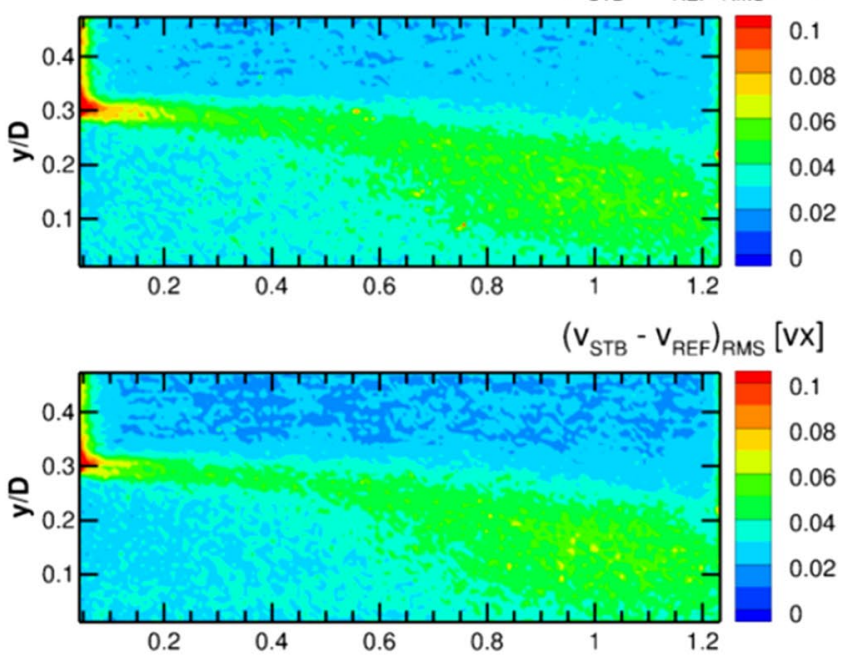

$\left(\mathrm{w}_{\mathrm{STB}}-\mathrm{w}_{\mathrm{REF}}\right)_{\mathrm{RMS}}[\mathrm{vx}]$

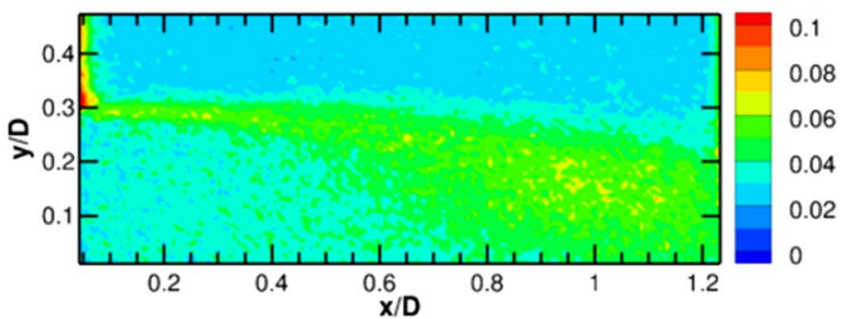

dle), and out-of-plane (bottom) directions. Note the difference in scale with respect to Fig. 7
Table 3 Overview of r.m.s. errors in streamwise velocity for different methods and regions of the flow

\begin{tabular}{|c|c|c|c|c|c|c|}
\hline \multirow[t]{3}{*}{ Processing } & \multicolumn{6}{|c|}{ Velocity error (vox. displacement) } \\
\hline & \multicolumn{2}{|c|}{ Freestream } & \multicolumn{2}{|c|}{ Reattachment region } & \multicolumn{2}{|c|}{ Global } \\
\hline & Clean & Noisy & Clean & Noisy & Clean & Noisy \\
\hline PIV (standard cross-corr.) & 0.03 & 0.05 & 0.35 & 0.40 & 0.23 & 0.27 \\
\hline PIV (FTEE) & - & - & - & - & 0.21 & 0.21 \\
\hline STB (time-resolved) & $<0.01$ & 0.02 & 0.02 & 0.05 & 0.01 & 0.04 \\
\hline STB (multi-pulse) & $<0.01$ & 0.02 & 0.02 & 0.05 & 0.02 & 0.04 \\
\hline
\end{tabular}

$\left(\mathrm{u}_{\mathrm{FILT}}-\mathrm{u}_{\mathrm{REF}}\right)_{\mathrm{RMS}}[\mathrm{vx}]$

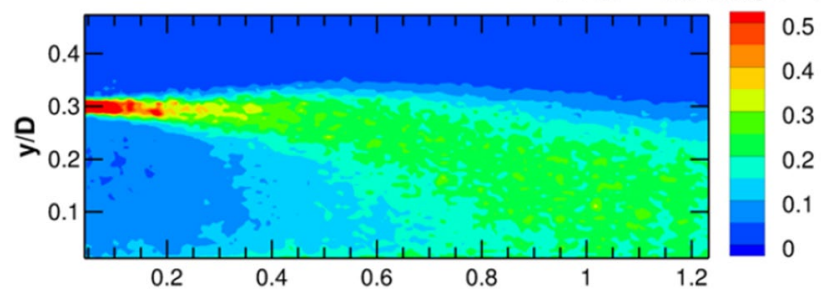

Fig. 9 Estimated contribution of spatial filtering associated with PIV to total error in the applied PIV processing (LaVision 2015). Figure 9 shows the result for a kernel size of 32 voxels, equivalent to the interrogation window size used in the present PIV processing.

Comparison with Fig. 7 shows that spatial filtering, indeed, makes a major contribution to the total error in the shear layer and reattachment region, e.g., for the clean case, the impact from filtering in the reattachment region is about 0.25 voxel displacement versus a total error of about 0.35 voxel. 


\section{Descriptions of applied pressure evaluation techniques}

\subsection{Flow modelling assumptions}

All methods rely on the momentum equation (Eq. 1) to determine the pressure gradient from the material acceleration. To account for compressibility effects, the momentum equation is rewritten to eliminate density as independent variable, under the assumption of inviscid and adiabatic flow (Eq. (6); van Oudheusden et al. 2007). The isentropic pressure ratio (Eq. 7) at the top of the domain is used as Dirichlet boundary condition for the spatial integration of the pressure gradient or to normalise the result of the integration procedure:

$\nabla \ln \left(\frac{p}{p_{\infty}}\right)=-\frac{1}{\mathrm{RT}} \cdot \frac{\mathrm{D} \mathbf{u}}{\mathrm{D} t}=-\frac{\gamma M_{\infty}^{2}}{V_{\infty}^{2}+\frac{\gamma-1}{2} M_{\infty}^{2}\left(V_{\infty}^{2}-V^{2}\right)} \cdot \frac{\mathrm{D} \mathbf{u}}{\mathrm{D} t}$,

$\frac{p}{p_{\infty}}=\left(1+\frac{\gamma-1}{2} M_{\infty}^{2}\left(1-\frac{V^{2}}{V_{\infty}^{2}}\right)\right)^{\frac{\gamma}{\gamma-1}}$.

The approximation of inviscid flow is motivated by the high Reynolds number. Using the simulation data, the value of the viscous term and its impact on the reconstructed pressure fields was, indeed, found to be at least two orders of magnitude smaller than that of the acceleration term (see Eq. 1). Figure 10 (top) shows the r.m.s. difference between adiabatic and reference temperature (top-left) and between pressure from isentropic relations and reference
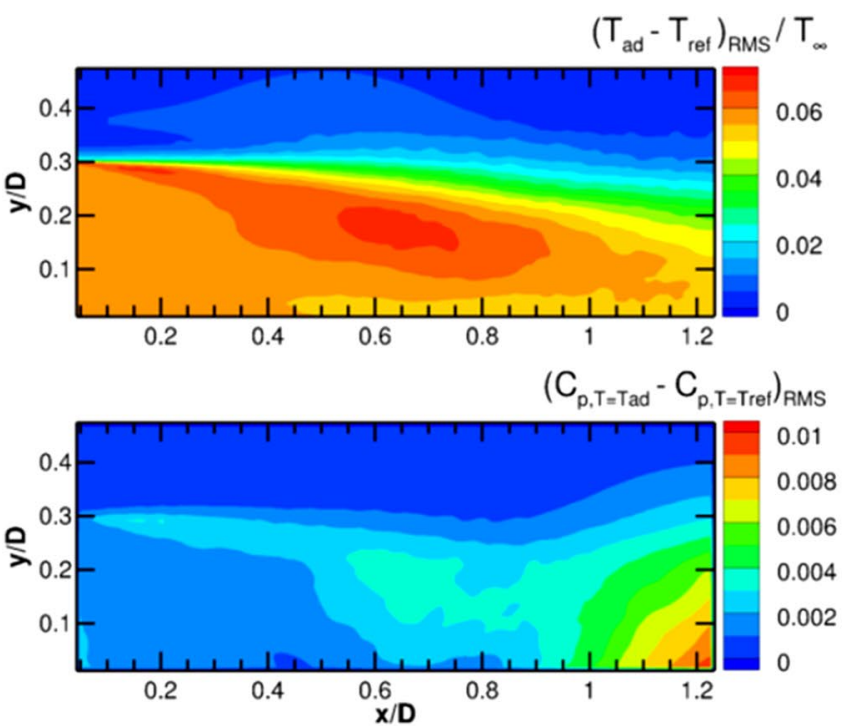

Fig. 10 R.m.s. difference between adiabatic and reference temperature (top-left); and between pressure from isentropic relations and reference pressure (top-right); impact of using adiabatic temperature pressure. The bottom figures show that the impact of using these flow modelling assumptions in the reconstruction of the pressure field is an order of magnitude smaller than the magnitude of the pressure fluctuations present in the flow (compare Fig. 4). The pressure fields used to perform the impact analysis were calculated similarly to the Eulerian approach described in Sect. 6.2 using simulation data interpolated to the PIV grid points as input data.

\subsection{PIV-based techniques}

The following four techniques reconstruct pressure fields from velocity data on a Cartesian grid, as obtained by PIV. The Eulerian approach (EUL) and (iterative) pseudoLagrangian approach (ILAG) require at least two subsequent snapshots as input data for determining the material acceleration (multi-snapshot approaches). Taylor's hypothesis approach (TH) and the instantaneous Vortex-in-Cell method (IVIC) require only a single velocity snapshot as input data (single-snapshot approaches).

\subsubsection{Iterative least-square pseudo-tracking (ILAG)}

In the pseudo-tracking approach, imaginary particles, initially located at the grid points, are tracked forward and backward in time using a series of PIV velocity fields (Liu and Katz 2006). Particle paths are determined by integration of the particle velocity which is obtained through linear spatial and temporal interpolation. The material acceleration is obtained from a first-order least-square fit through the velocity values at the positions of the

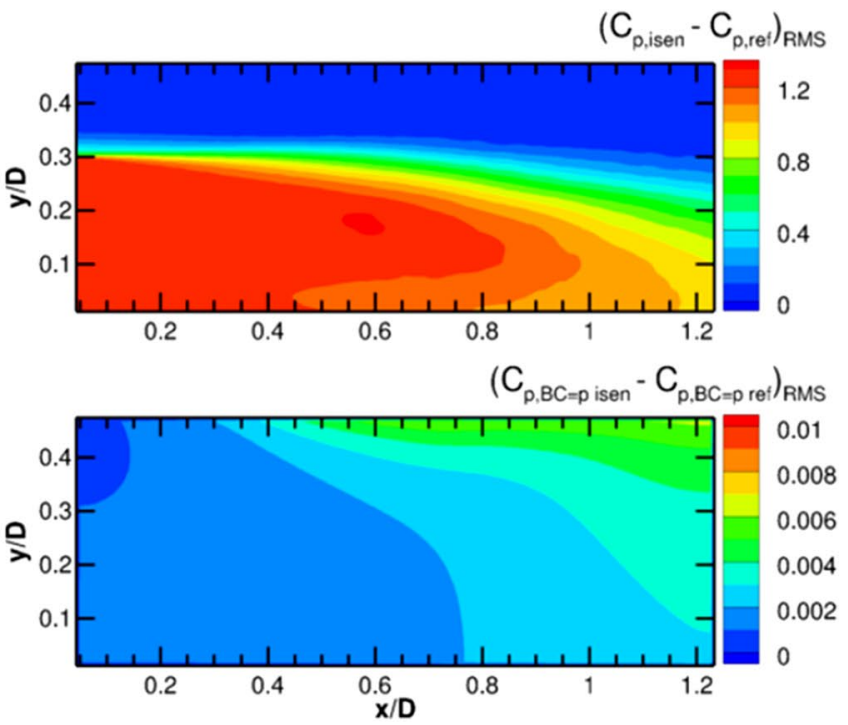

instead of reference temperature (bottom-left); impact of using pressure from isentropic relations instead of reference pressure as boundary condition at top wall (bottom-right) 
imaginary particles, disregarding any particle positions outside the domain. For time-resolved PIV, it could also be estimated with the FTEE algorithm (Jeon et al. 2014). The material acceleration is used to obtain a better estimate of the imaginary particle track in a next iteration. The temporal length of each trajectory is 9 time-steps for the case of time-resolved input data and 2 time-steps for the case of multi-pulse input data. To compensate the excluded outward fluid motion at the volume boundary and to prevent corresponding decrement of the dynamic range, the temporal trajectory length is extended toward the opposite side. The spatial integration of the pressure gradient is conducted using staggered grid by minimisation of a cost function. Neumann boundary conditions are imposed implicitly. A Dirichlet boundary condition with a value of zero is prescribed in a single grid point. In a next step, the solution is offset using the isentropic pressure ratio (see Eq. 7) sampled over a region selected based on the Frobenius norm of the material acceleration tensor. A detailed description of the present implementation can be found in (Jeon et al. 2015). The method is implemented using velocity fields from single-pair cross-correlation analyses, FTEE velocity data, as well as STB input data. In case of the latter, the temporal length is 2 time-steps for both the cases of timeresolved and multi-pulse input.

\subsubsection{Eulerian approach (EUL)}

The material acceleration is evaluated in a stationary reference frame as being composed of temporal and spatial velocity derivatives (see Baur and Köngeter 1999). In the present implementation, these derivatives are evaluated using the second-order finite difference discretization except at the boundaries where the first-order discretization is used instead. The logarithmic of the pressure gradient is integrated by first taking the divergence of Eq. (6) to obtain a Poisson equation (see de Kat and van Oudheusden 2012). Neumann boundary conditions are imposed on all sides except for the top surface of the domain, where the pressure as obtained from the isentropic flow relations is prescribed as Dirichlet boundary condition (see Eq. 7). The problem is discretised using a second-order finite difference scheme and the resulting linear system is subsequently solved for pressure. The method is implemented using pairs of snapshots with a time separation of $10 \mu$ s both for the multipulse and the time resolved case.

\subsubsection{Taylor's hypothesis approach $(\mathrm{TH})$}

Taylor's hypothesis consists of the assumption that advection due to turbulent circulation is small and that 'frozen' turbulence, therefore, advects with the flow. Adopting Taylor's hypothesis enables the estimation of the material acceleration from a single velocity snapshot. The choice of convection velocity is crucial for the accuracy of this method and is taken here as the mean velocity, averaged in z-direction. A detailed description of the implementation of this approach for pressure determination can be found in de Kat and Ganapathisubramani (2013) and Laskari et al. (2016). The subsequent integration of the pressure gradient is performed similarly as for the Eulerian approach described above.

\subsubsection{Instantaneous Vortex-in-Cell (IVIC)}

In the IVIC method, the material acceleration is approximated from a single snapshot by application of the vorticity transport equation to the velocity measurement at a single time instant. In summary, the method first evaluates the vorticity temporal derivative by solution of the inviscid, incompressible vorticity transport equation. In a next step, the velocity temporal derivative is calculated by solution of a Poisson equation and then used to construct the material acceleration. A more detailed description of the approach can be found in Schneiders et al. (2016). The subsequent integration of the pressure gradient is performed similarly as for the Eulerian approach described above.

\subsection{LPT-based techniques}

The following four techniques reconstruct pressure fields from acceleration data along particle tracks. FlowFit A and VIC + first interpolate acceleration data to a Cartesian grid and subsequently solve the momentum equation for pressure. FlowFit B and Voronoi-based integration directly determine pressure fields from the sparse acceleration data.

\subsubsection{FlowFit A (FFA)}

The acceleration data obtained at the particle positions are interpolated to a Cartesian grid by 'FlowFit'; an iterative optimization approach that generates a quadratic B-splines representation of the acceleration field, using spline coefficients from minimizing a cost function that enforces smoothness and penalizes the curl of acceleration (Gesemann et al. 2016). Integration is performed in Fourier space (Huhn et al. 2016). A constant pressure offset is added to the solution to yield the absolute pressure field.

\subsubsection{FlowFit B (FFB)}

Flowfit B represents the scalar pressure field as 3D cubic B-spline function and estimates its parameters by defining a cost function that is minimized on the basis of a weighted sum of: 
- Squared distances between "fitted" and "measured" pressure gradients at all particle positions.

- Squared distances between "fitted" and isentropic pressure at the top of the domain.

- Squared curvatures of the pressure function as a form of Tikhonov regularization.

The resulting linear least-squares problem is solved iteratively. After this reconstruction, the B-spline curve is sampled at the desired points. Due to doubts about the impact of compressibility effects in the subsonic compressible flow, for both FFA and FFB, it was decided not to impose zero-divergence of the velocity field and thus not to take advantage of a non-linear regularization approach, in contrast to the VIC+ method. The full version of the FlowFit algorithm, however, does include a complete non-linear Navier-Stokes regularization (similar to VIC+) and is described in Gesemann et al. (2016).

\subsubsection{Vortex-in-Cell+ (VIC+)}

VIC+ reconstructs instantaneous velocity and its material derivative on a regular grid using both the instantaneous velocity and material acceleration evaluated from Lagrangian particle trajectory measurements. The technique follows an iterative procedure that minimizes a cost function and penalizes deviations from the instantaneous velocity and material acceleration measurement. A detailed description of the approach can be found in Schneiders and Scarano (2016). The subsequent integration of the pressure gradient is performed via a Poisson equation (see Eulerian approach). Pressure gradients are prescribed as Neumann boundary conditions used on all sides of the domain. In a next step, the solution is normalised by setting the mean pressure ratio at the top plane equal to the mean isentropic pressure ratio [see Eq. (7)].

\subsubsection{Voronoi-based pressure integration (VOR)}

A Lagrangian finite-volume method is utilized to spatially integrate Eq. (6) to obtain pressure values at the particle positions provided by STB. First, a Lagrangian network is constructed on the particle field (Neeteson and Rival 2015; Neeteson et al. 2016), so that the pressure may be extracted directly in the Lagrangian frame. Next, Eq. (6) is discretized to the Lagrangian network using a finite-volume method. Neumann boundary conditions are enforced implicitly at all sides excluding the top where a Dirichlet boundary condition is enforced by prescribing the isentropic pressure ratio (see Eq. 7). The resulting problem is expressed in a system of linear equations, which is solved for pressure.

\section{Pressure results}

The reconstructed pressure fields are compared to reference pressure field from the simulation data and to each other. All reconstructed pressure fields are given for the same grid size as the PIV velocity data, i.e., $171 \times 67 \times 11$ data points, with the exception of VOR which is based on $165 \times 61 \times 6$ data points. For the time-resolved case, 36 snapshots are considered with exception of ILAG applied to FTEE velocity input data for which only 29 pressure snapshots are available. The result for the multi-pulse case consists of 21 time instances.

\subsection{Pressure from PIV-based techniques}

Figures 11 and 12 show the (reconstructed) pressure fields and their errors calculated from PIV time-resolved and multi-pulse input data, respectively. All results pertain to the noisy case. The results for the clean case are very similar and have, therefore, been omitted for brevity. Reference pressure from the simulation data is shown in the top-left figure. Each other row corresponds to a different technique. Left figures show a sample of the instantaneous (reconstructed) pressure fields in the centre-plane, with the corresponding instantaneous errors in the centre figures. Right figures show the r.m.s. errors from all available time-steps and all planes in $z$-direction. As the error of the reconstructed pressure fields generally increase towards the outer planes, this approach increases the r.m.s. error level with respect to the error at centre $z$-plane. The overall comparison is however not affected. The reference pressure is provided for the same time instance as that of ILAG and EUL, which is $5 \mu$ s later than the depicted time instance for $\mathrm{TH}$ and IVIC.

The left figures show that all methods are able to reconstruct the general features of the reference pressure field. The centre and right figures show that all reconstructed pressure fields have the highest error in the shear layer and reattachment region where the smallest temporal and spatial flow scales occur and the precision of the PIV measurement is poorest (see Fig. 7).

Overall, the most accurate reconstructed pressure fields are obtained with ILAG (compare right figures). For the case of time-resolved input data, the use of more accurate FTEE velocity data (see Sect. 5.3) significantly improves the quality of the reconstructed pressure field with respect to standard PIV cross-correlation. Investigation of the time-resolved sequence of instantaneous results shows highly fluctuating error fields for the Eulerian approach (EUL), whereas the error field for ILAG evolves more gradually over time. This is attributed to the better use of temporal information by ILAG, which in the present implementation for the time-resolved fits 

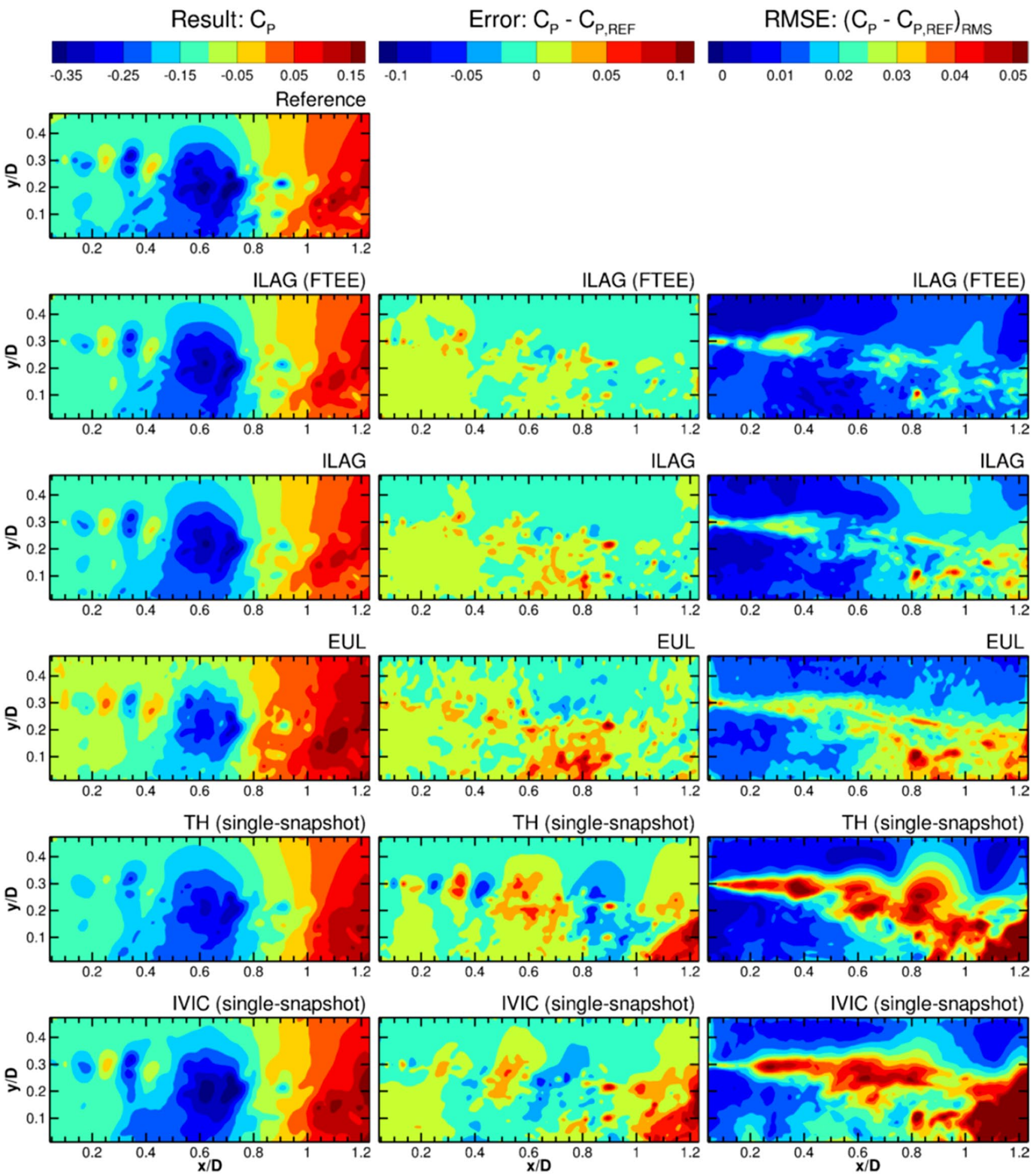

Fig. 11 Results and errors from applying PIV-based pressure field reconstruction techniques to time-resolved input data. (Reconstructed) instantaneous pressure fields in the centre-plane (left col-

a second-order polynomial over 9 consecutive velocity fields. The temporal velocity derivative in the current Eulerian approach on the other hand is based on the umn), instantaneous error in the centre-plane (centre column), and r.m.s. errors for all z-planes (right column)

difference between two velocity fields, a procedure that is known to be sensitive to any velocity measurement noise.

For the case of the multi-pulse input data, the difference between the results of ILAG and EUL is less 

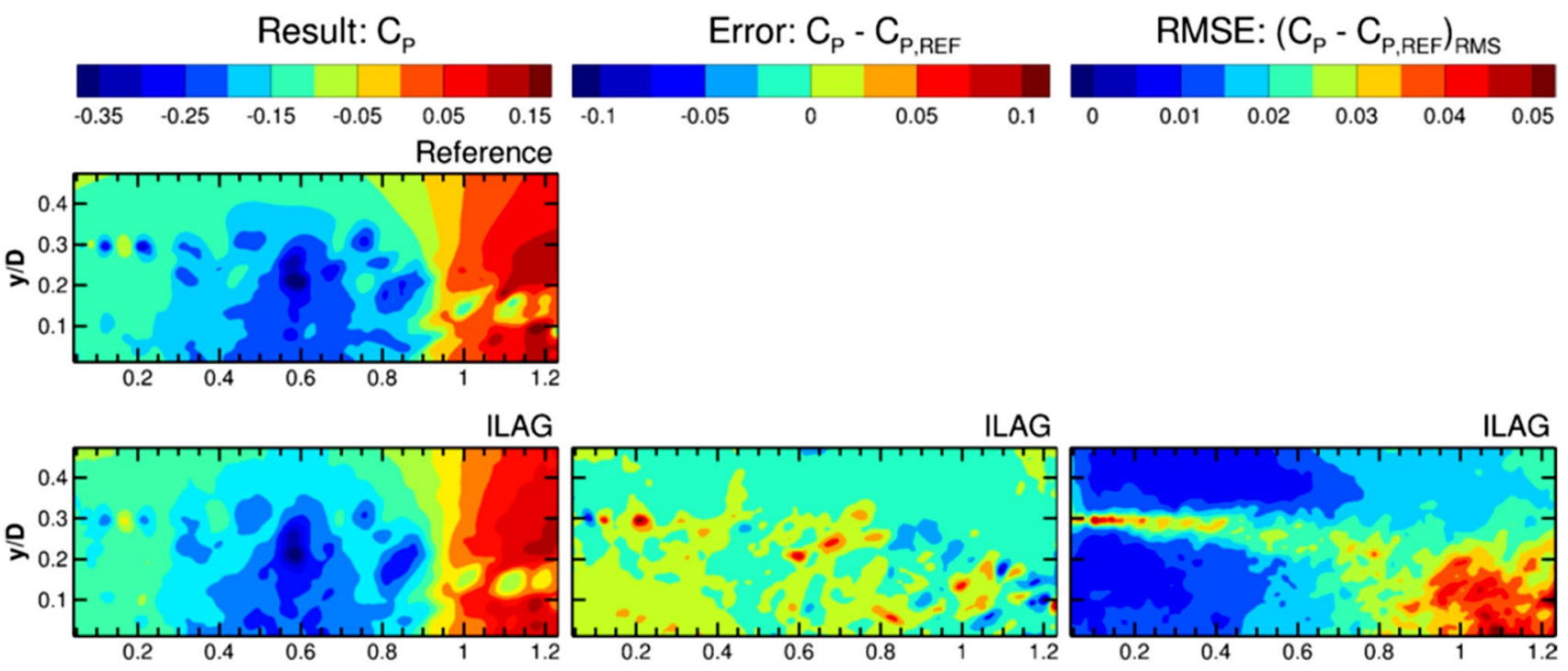

ILAG

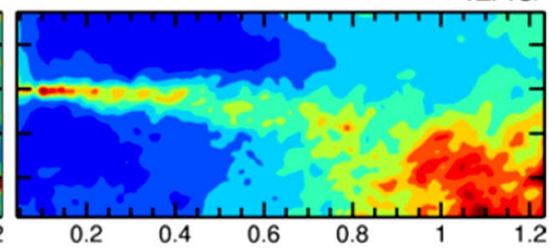

EUL
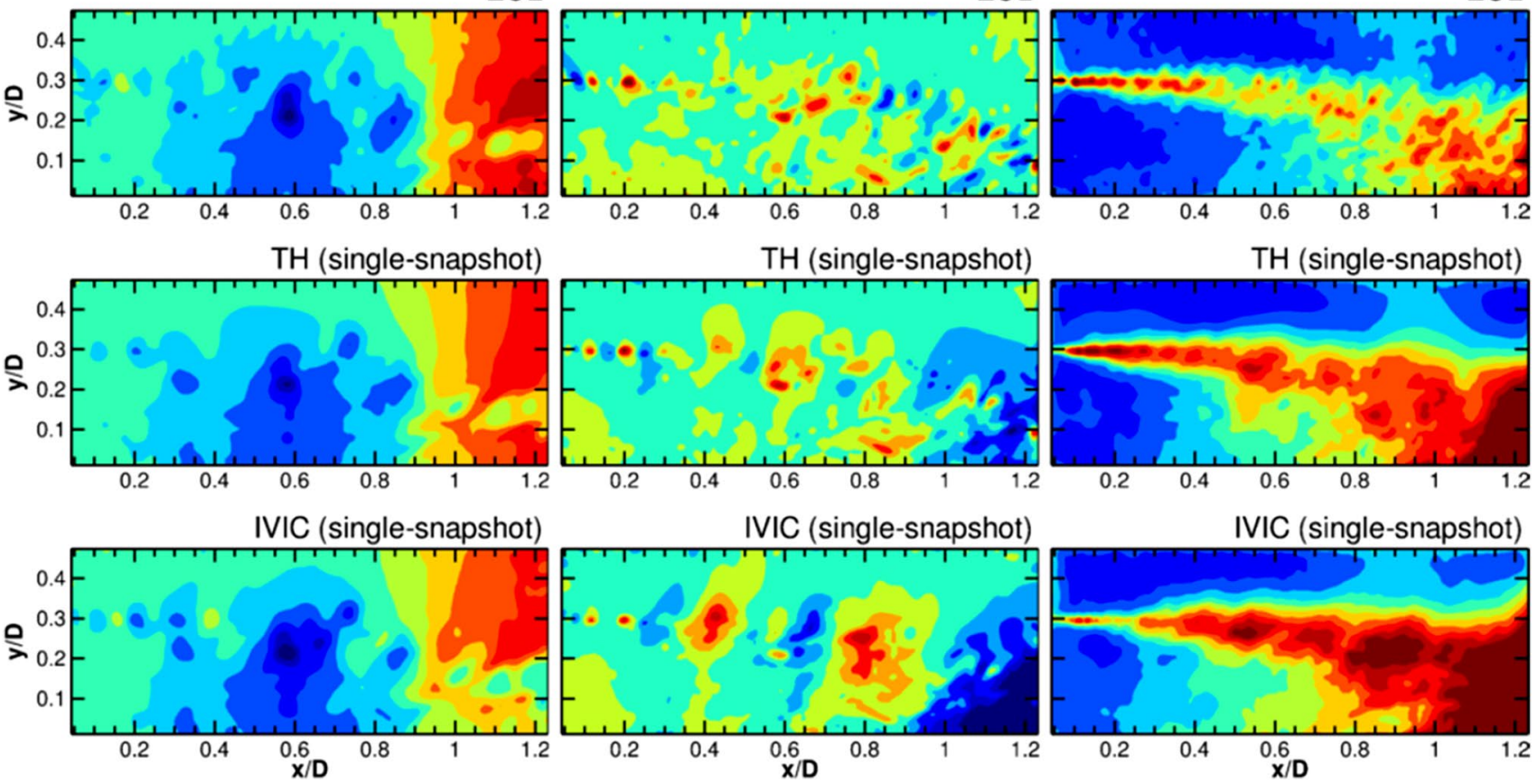

Fig. 12 Results and errors from applying PIV-based pressure field reconstruction techniques to multi-pulse input data. (Reconstructed) instantaneous pressure fields in the centre-plane (left column), instantaneous error in the centre-plane (centre column), and r.m.s. errors for all z-planes

pronounced as both only use two velocity snapshots. Here, the slightly lower overall error levels of ILAG may speculatively be attributed to a number of possible causes including the filtering effect of making a polynomial fit, better numerical treatment of regions near the domain boundary, and a more suitable time separation between velocity fields. Further comparison shows that the r.m.s. errors for ILAG are relatively high in the top-right of the domain. This is attributed to difference in the boundary condition for pressure integration as contrary to the other methods, as the present implementation of ILAG does not prescribe a pressure ratio at the downstream part of the top boundary.

The single-snapshot approaches (TH and IVIC) can be seen to produce less precise reconstructed pressure fields than the multi-snapshots approaches (ILAG and EUL); an expected result as EUL and ILAG use more temporal information. Nevertheless, in the light of practical difficulties in obtaining multi-pulse data in high-speed flows, it is encouraging that from this assessment, it appears to be quite feasible to reconstruct the main pressure field features from a single velocity snapshot. 
Comparison of the single-snapshot approaches (TH and IVIC) shows that IVIC better captures the smaller flow structures in the upstream part of the shear layer (for $x / D<0.3)$. In the rest of the domain, TH performs better. The performance of $\mathrm{TH}$ here is surprisingly good as it was designed to work for convective flows and the underlying assumption of advection of 'frozen' turbulent structures is invalid for shear layers and separated flows. The errors of IVIC in the downstream region are likely due to the difficulty in defining a suitable boundary condition for the velocity temporal derivative in this region, which is inherent to the method and a known sensitive aspect of its implementation for thin-volume tomographic PIV.

In Sect. 5.3, it was shown that the velocity error from PIV for a large part is due to the inherent spatial filtering. To assess the contribution of spatial filtering to the pressure error, Fig. 13 shows the difference between the reference pressure solution and a spatially filtered solution, obtained using a similar approach as outlined in Sect. 5.3. The figure shows that for pressure, contrary to the situation for velocity, the contribution of filtering to the total error is relatively small.

\subsection{Pressure from LPT-based techniques}

Figures 14 and 15 show the (reconstructed) pressure fields and their errors for LPT time-resolved and multi-pulse noisy input data, respectively. Note that the scaling of the instantaneous error (centre figures) has been adapted with respect to Sect. 7.1 to better visualize the error distribution. The scaling of r.m.s. error fields (right figures) remains unchanged to facilitate direct comparison with the PIVbased techniques.

The left figures show that all LPT-based techniques are able to reconstruct the general features of the reference pressure field and that, moreover, most methods provide similar results, especially for the time-resolved data (Fig. 14). Errors for the time-resolved input data are lower than for the multi-pulse input data, which is attributed to lower accuracy of the particle tracks in the latter case.

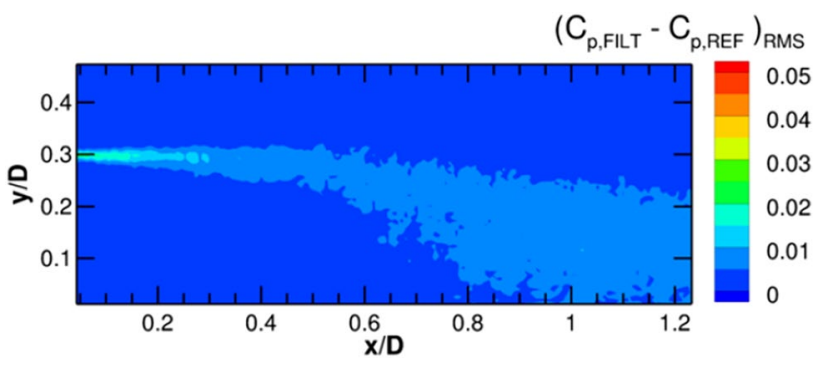

Fig. 13 Estimated contribution of spatial filtering associated with PIV to total error
Comparison of results of the different techniques shows that the results for FlowFit A and FlowFit B are very similar and VIC+ yields the most accurate reconstructed pressure fields. Errors for the Voronoi-based integration approach (VOR) are significantly higher than for the other methods.

\subsection{Reconstruction of pressure length scales}

The reconstruction of the pressure length scales present in the flow is analysed by considering spatial spectra along horizontal gridlines (see Fig. 16). The ZDES simulation data are first interpolated to the same grid points as used for the pressure results. Spectra are obtained by applying the FFT algorithm after using the symmetric straight line approach as outlined in Foucaut et al. (2004) and without any windowing function, similarly to the approach used in Liu and Katz (2013). The wavelength is scaled by the interrogation window size used in the PIV correlation analysis, which is equal to four times the grid spacing. Statistical convergence has been improved by averaging the spectra from all time instances and from 40 horizontal gridlines between the afterbody and the shear layer $(0.03>y / D>0.30)$. The spectra are shown for frequencies up to the Nyquest frequency based on the interrogation window size: $2 \lambda=\mathrm{WS}$.

The spectra for the PIV-based pressure field reconstructions (left figure) show that ILAG, EUL, and IVIC all reconstruct the reference pressure spectrum for large-scale pressure fluctuations $(\mathrm{WS} / \lambda<0.2)$. In addition, the spectra for ILAG, EUL, and IVIC are very similar for $\mathrm{WS} / \lambda<0.35$. The spectrum for Taylor's hypothesis in this spectral band is much lower. This discrepancy is to be expected as the hypothesis does not hold for large-scale structures. For wavelengths $\mathrm{WS} / \lambda>0.35$, the spectra for ILAG, EUL, and $\mathrm{TH}$ all lie below the reference spectrum, indicating that these approaches do not fully reconstruct small-scale fluctuations. Comparison of the results for ILAG show that the use of FTEE input data brings its spectrum closer to the reference spectrum suggesting better reconstruction of smallspace structures. The spectrum for the Eulerian approach (EUL) much better follows the reference spectrum than those of the other approaches, despite its r.m.s. error being similar to ILAG (compare Fig. 11).

The spectra for the LPT-based pressure field reconstructions (right figure) show that all tested approaches follow the reference pressure spectrum for $\mathrm{WS} / \lambda<0.2$. For smaller wavelengths, FFA, FFB, and VIC+ better reconstruct the reference spectrum than ILAG and VOR. All methods at some point cross the reference spectrum indicating a spectral noise floor. The noise floor for VOR is relatively high and that of VIC+ is the lowest, which is consistent with earlier observations of the error levels (compare Figs. 14, $15)$. 

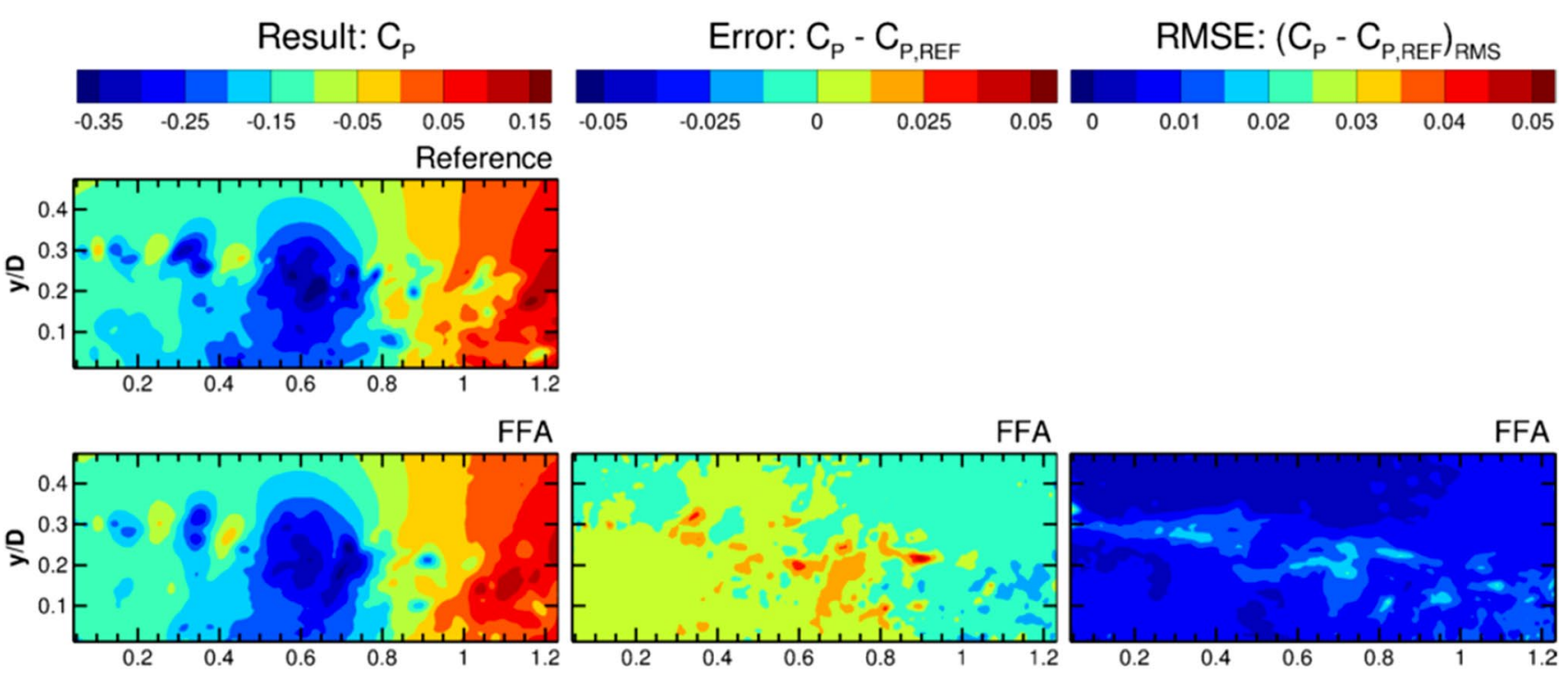

FFA

FFA
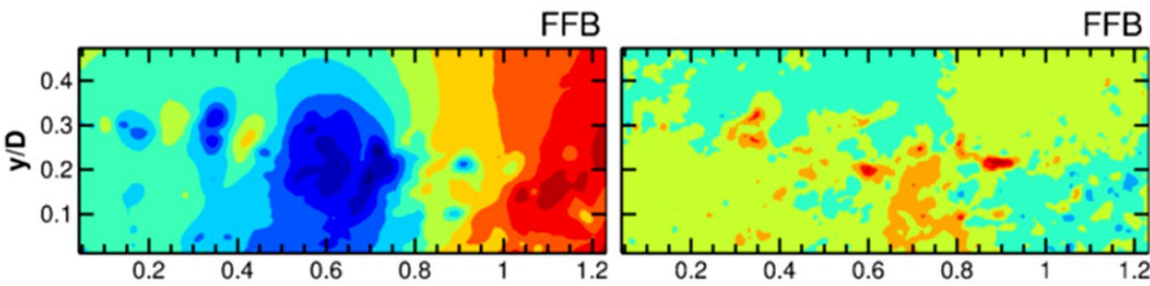

FFB
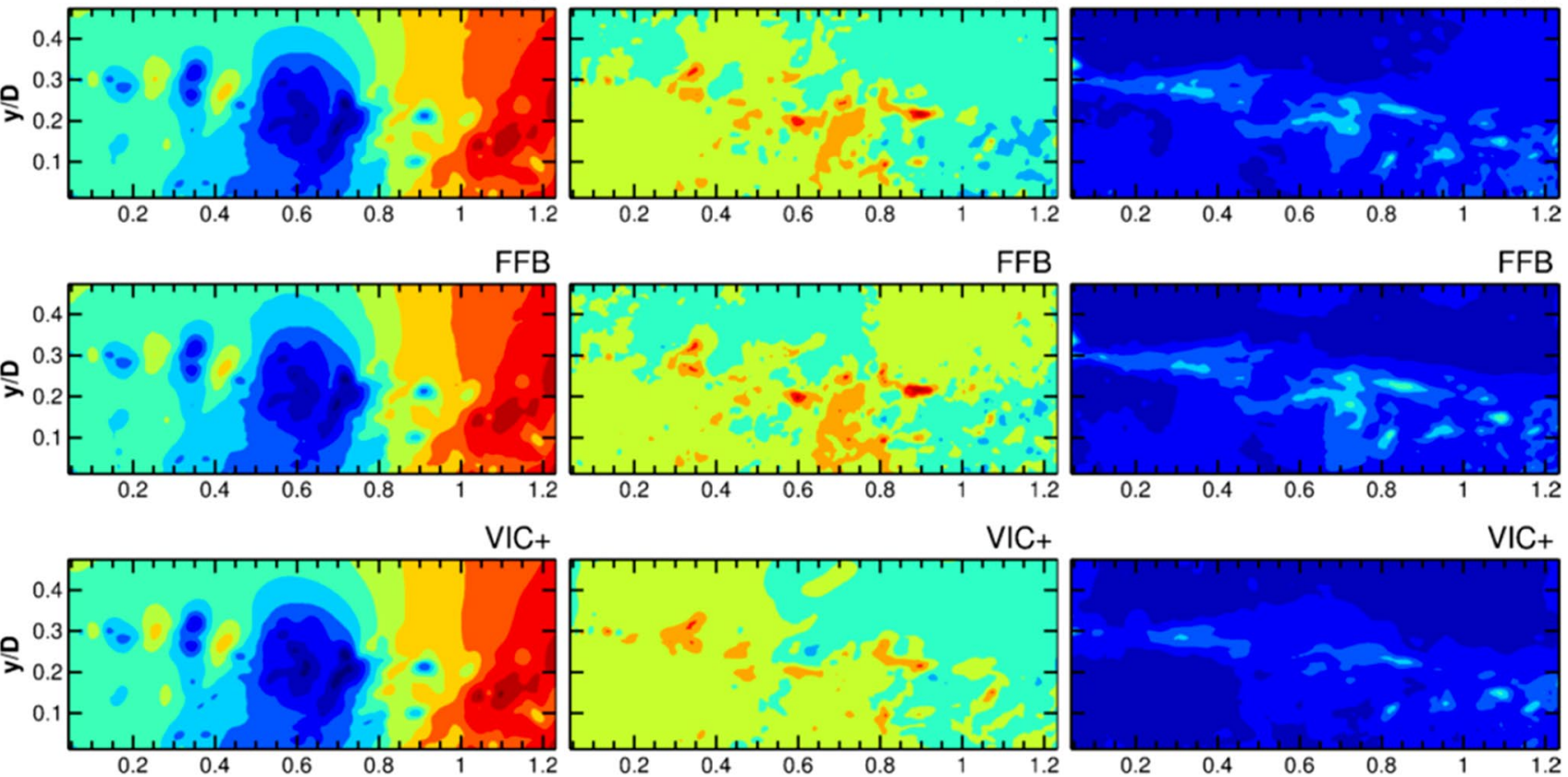

FFB
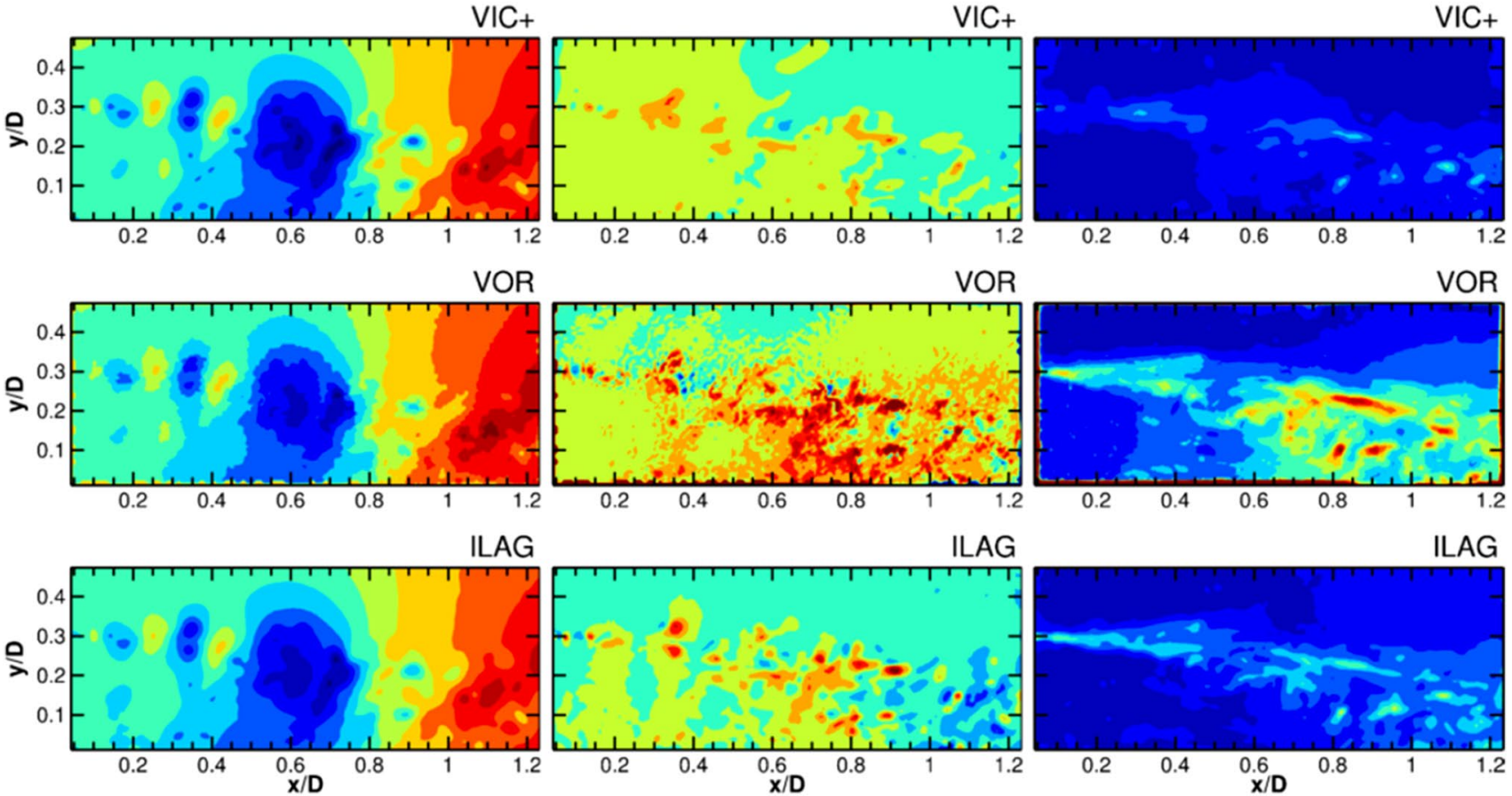

Fig. 14 Results and errors from applying LPT-based pressure field reconstruction techniques to time-resolved input data. (Reconstructed) instantaneous pressure fields in the centre-plane (left col-

umn), instantaneous error in the centre-plane (centre column), and r.m.s. errors for all z-planes (right column)

Comparing PIV- and LPT-based approaches shows that both allow reconstruction of the spectrum for largescale pressure fluctuations. The LPT-based approach, however, better reconstructs the spectrum for small-scale fluctuations. Contrary to the LPT-based approaches, the PIV-based approaches do not exhibit a clear noise floor. It 

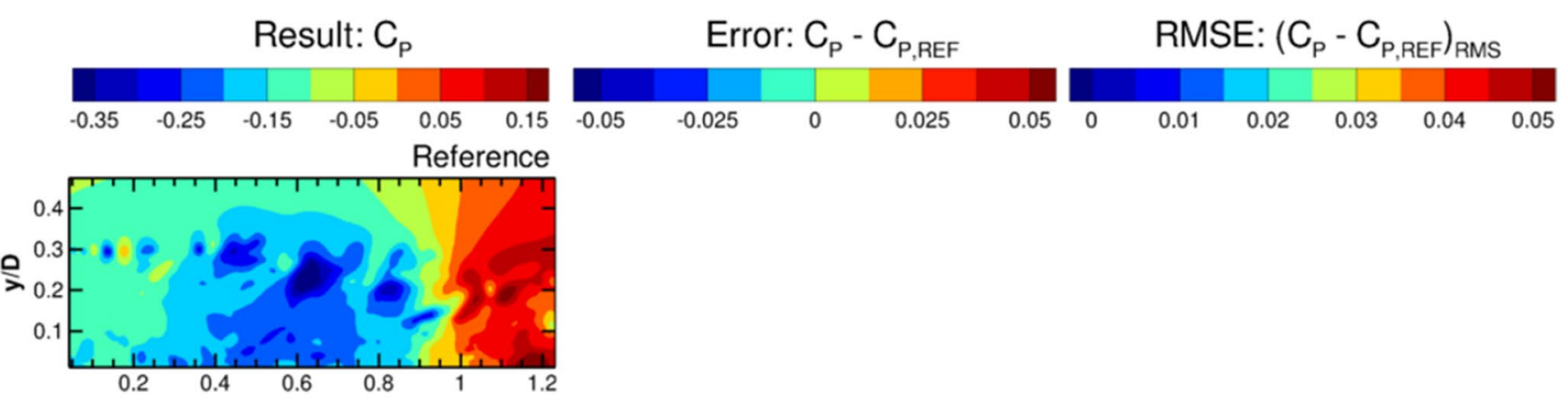

FFA
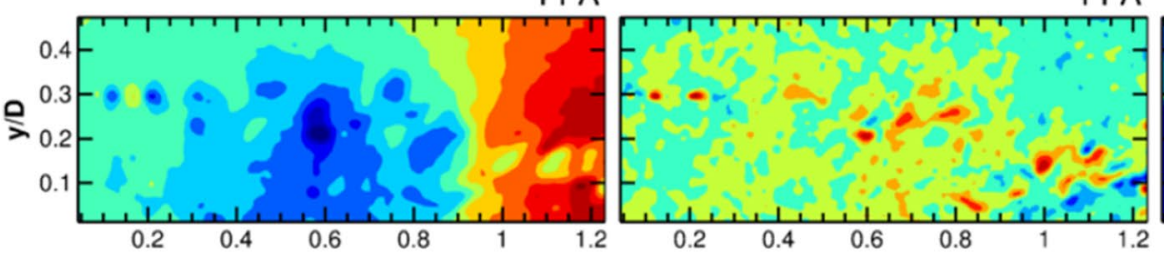

FFA

FFA

FFB
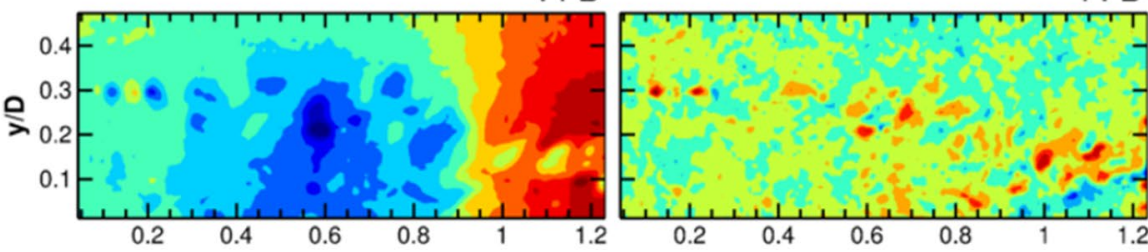

FFB
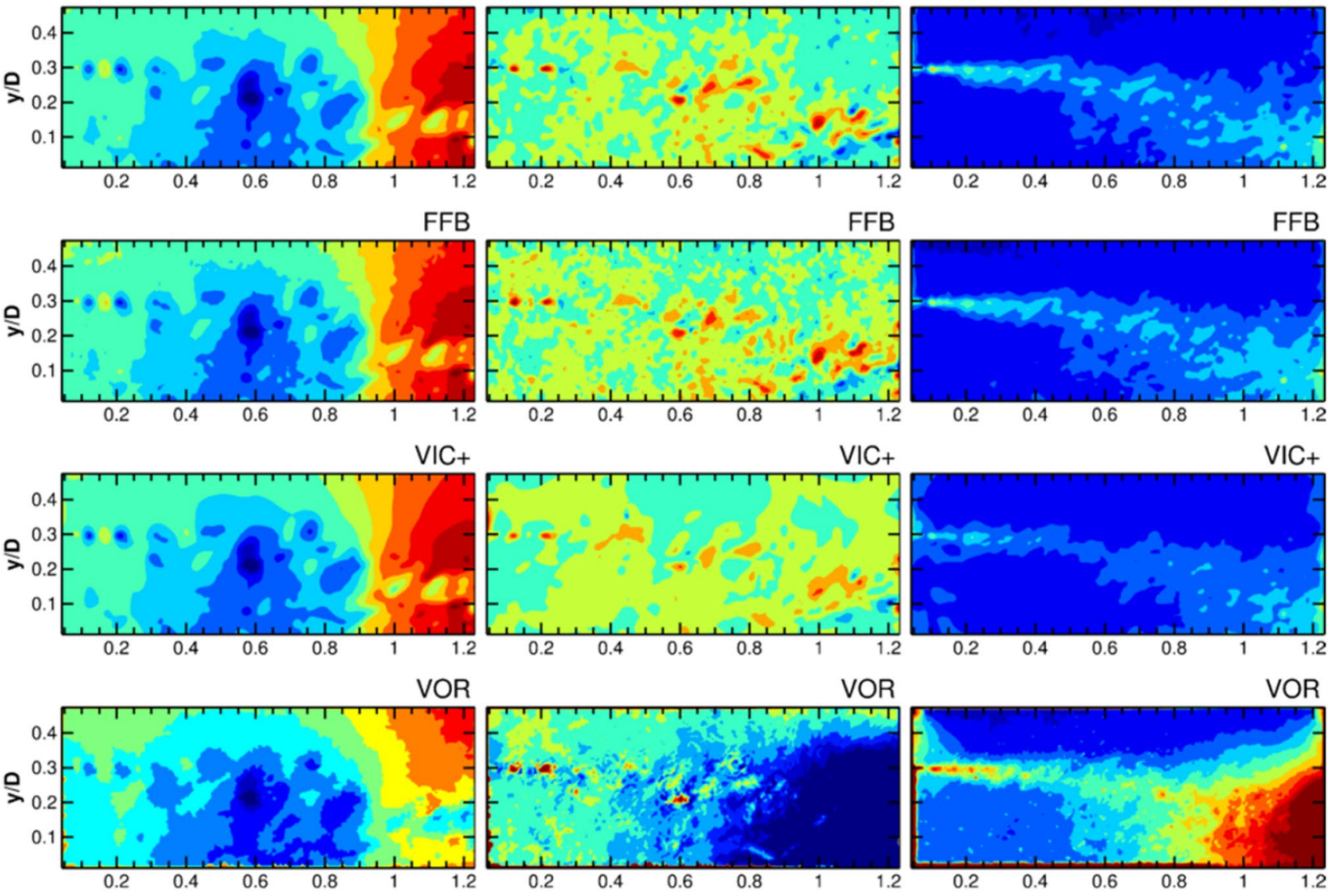

ILAG

ILAG
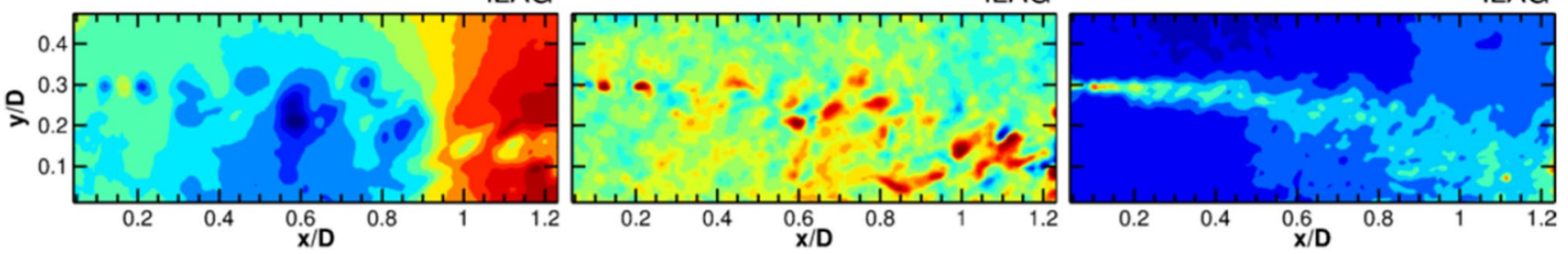

Fig. 15 Results and errors from applying LPT-based pressure field reconstruction techniques to multi-pulse input data. (Reconstructed) instantaneous pressure fields in the centre-plane (left column), instan-

taneous error in the centre-plane (centre column), and r.m.s. errors for all z-planes (right column)

is speculated that the spatial filtering of the velocity field due to the PIV processing also leads to filtering of smallscale noise in the reconstructed pressure fields.

\subsection{Global pressure error}

To further quantify and compare the performance of different methods, the global pressure errors $\left(\varepsilon_{\mathrm{RMS}}^{\text {global }}\right)$ is obtained 

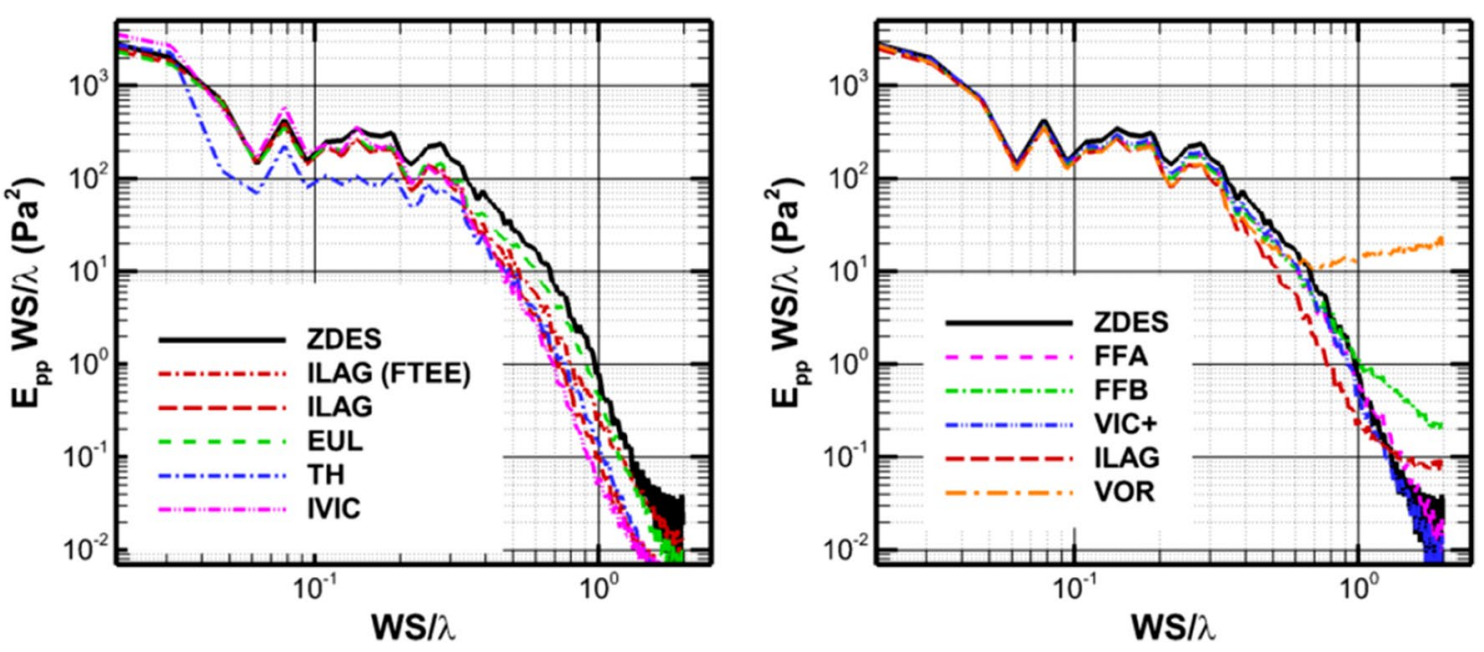

Fig. 16 Spatial spectra of pressure fluctuations along 40 horizontal lines between the afterbody and the shear layer $(0.03>y / D>0.30)$ for PIVbased pressure field reconstructions (left) and LPT-based pressure field reconstructions (right)

by taking the r.m.s. error for all gridpoints $\left(\mathrm{N}_{\mathrm{p}}\right)$ at all time instances $\left(\mathrm{N}_{\mathrm{t}}\right)$ :

$\varepsilon_{\mathrm{RMS}}^{\text {global }}=\sqrt{\frac{1}{\mathrm{~N}_{\mathrm{t}} \mathrm{N}_{\mathrm{p}}} \sum_{i=1}^{\mathrm{N}_{\mathrm{t}}} \sum_{j=1}^{\mathrm{N}_{\mathrm{p}}}\left(\left(\mathrm{C}_{\mathrm{p}}\right)_{i, j}-\left(\mathrm{C}_{\mathrm{p}, \mathrm{ref}}\right)_{i, j}\right)^{2}}$.

Table 4 shows the resulting errors for all cases considered. The methods have been ordered per input data and for increasing errors. The global error for FTEE+ ILAG has been scaled as described in Sect. 5.3.

Errors for the noisy case can be seen to be slightly higher than for the clean case. Where this is not the case, this is attributed to a lack of statistical convergence. The results for the clean and noisy case follow a similar trend which suggests that none of the methods is critically sensitive to the amount of noise added in this test case. LPTbased approaches result in significantly lower error levels than the PIV-based approaches. This is in line with the lower velocity errors from STB compared to PIV for the present test case (see Sect. 5.3). Another indication that the lower errors are driven by the better input data is that the ILAG approach performs much better when using LPT data than when using PIV data.

To put the magnitude of the error levels in perspective, it is useful to note that the error in the table is expressed in $0.01 \mathrm{C}_{\mathrm{p}}$ which is equivalent to a percentage of the freestream dynamic pressure. Furthermore, with the global level of pressure fluctuations $\left(\left(\mathrm{C}_{\mathrm{p}}^{\prime}\right)_{\mathrm{RMS}}^{\text {global }}\right)$ being $4 \%$ of the local dynamic pressure (see Eq. 9), it follows that a $1 \%$ error level in the table corresponds to $25 \%$ of the global pressure fluctuations. The limited number of snapshots does not allow to make a meaningful decomposition of this error into a bias and random part:

$$
\left(\mathrm{C}_{\mathrm{p}}^{\prime} \mathrm{p}_{\mathrm{RMS}}^{\text {global }}=\sqrt{\frac{1}{\mathrm{~N}_{\mathrm{t}} \mathrm{N}_{\mathrm{p}}} \sum_{i=1}^{\mathrm{N}_{\mathrm{t}}} \sum_{j=1}^{N_{\mathrm{p}}}\left(\left(\mathrm{C}_{\mathrm{p}, \text { ref }}\right)_{i, j}-\left(\mathrm{C}_{\mathrm{p}, \text { ref,mean }}\right)_{j}\right)_{i, j}^{2}}=0.04 .\right.
$$

Table 4 Global pressure errors for all noisy and clean, timeresolved (TR), and multi-pulse (MP) input data

\begin{tabular}{llllll}
\hline \multirow{2}{*}{$\begin{array}{l}\text { Pressure field } \\
\text { reconstruction }\end{array}$} & Source of input data & \multicolumn{4}{l}{ Global pressure error $\left(10^{-2}\right)$} \\
\cline { 3 - 5 } & & TR, clean & TR, noisy & MP, clean & MP, noise \\
\hline VIC+ & LPT & 0.68 & 0.63 & 0.71 & 1.02 \\
FFA & LPT & 0.71 & 0.74 & 0.82 & 1.07 \\
FFB & LPT & 0.72 & 0.76 & 0.86 & 1.10 \\
ILAG & LPT & 0.88 & 0.89 & 0.99 & 1.23 \\
VOR & LPT & 1.71 & 1.63 & 3.57 & 2.35 \\
ILAG & PIV (FTEE) & 1.45 & 1.33 & - & - \\
ILAG & PIV & 1.43 & 1.69 & 1.67 & 2.10 \\
EUL & PIV & 1.52 & 2.09 & 1.80 & 2.11 \\
TH & PIV (1 snapshot) & 2.23 & 2.54 & 2.66 & 2.78 \\
IVIC & PIV (1 snapshot) & 3.44 & 2.99 & 3.34 & 3.39 \\
\hline
\end{tabular}




\section{Conclusions}

A test case for PIV-based and LPT-based pressure evaluation techniques has been developed by constructing a simulated experiment from ZDES simulation data. Important experimental error sources are replicated by simulating the entire measurement chain. To enable an assessment of the impact of measurement noise, the test case includes both cases with and without added artificial noise. The test case includes multi-pulse data (4 particle images; 2 velocity snapshots), which is representative of the acceleration measurement procedure available in high-speed flows, as well as longer series of continuous time-resolved data, which can realistically only be obtained for low-speed flows. Both tomographic PIV algorithms and the STB algorithm have been applied to enable the testing of both PIVand LPT-based pressure determination procedures.

A variety of state-of-the-art pressure determination techniques has been applied to a single test case consisting of a high-speed, subsonic compressible flow over an axisymmetric step. The working principles as well as the implementation of these techniques have been described. Using the test case, the techniques have been assessed in terms of their capability to reconstruct the reference pressure field as well as the impact of noise in the input data and the benefit of using time-resolved data. This led to the following main conclusions

1. A range of suitable methods exists that can reconstruct instantaneous pressure fields from PIV/LPT input data. The main features of the pressure fields can be reconstructed from a single PIV velocity snapshot while highly accurate pressure fields can be reconstructed using STB in combination with more advanced techniques.

2. For the present input data, the LPT-based techniques produce more accurate pressure fields than the PIVbased approaches in terms of magnitude as well as length scales. This difference in performance is attributed to a combination of higher spatial resolution of the input data and better use of time information in the data sets.

3. The use of longer series of time-resolved input data allows more accurate reconstructed pressure fields. Nevertheless, in light of practical difficulties in obtaining multi-pulse data for high-speed flows, it is encouraging that it appears to be possible to reconstruct the main features of the reference pressure field already from a single velocity field.

4. Noise in the input data typically reduces the accuracy of the reconstructed pressure fields, but none of the methods proved to be critically sensitive to the amount of noise that was added in the present test case.
Given these conclusions on the availability and performance of PIV/LPT-based pressure field reconstruction algorithms and their dependence on the availability of suitable experimental data, future advances are best attained through demonstration through experiments and further development of experimental capabilities.

Acknowledgements This work is supported by the European FP-7 project "NIOPLEX", grant agreement 605151. The authors would like to thank Guillaume Gomit from Institut PPRIME for applying the FTEE algorithm.

Open Access This article is distributed under the terms of the Creative Commons Attribution 4.0 International License (http:// creativecommons.org/licenses/by/4.0/), which permits unrestricted use, distribution, and reproduction in any medium, provided you give appropriate credit to the original author(s) and the source, provide a link to the Creative Commons license, and indicate if changes were made.

\section{References}

Adrian RJ, Yao C-S (1985) Pulsed laser technique application to liquid and gaseous flows and the scattering power of seed materials. Appl Opt 24:44. doi:10.1364/AO.24.000044

Atkinson C, Soria J (2009) An efficient simultaneous reconstruction technique for tomographic particle image velocimetry. Exp Fluids 47:553-568. doi:10.1007/s00348-009-0728-0

Baur T, Köngeter J (1999) PIV with high temporal resolution for the determination of local pressure reductions from coherent turbulence phenomena. 3rd Int Work Part Image Velocim, Santa Barbara

Blinde PL, Lynch KP, van Oudheusden BW, Schneiders JFG, Schrijer FFJ (2014) Assessment of instantaneous pressure determination in a transonic base flow based on four-pulse tomographic PIV. 17th Int Symp Appl Laser Tech to Fluid Mech

Blinde PL, Lynch KP, van Oudheusden BW, Schrijer FFJ (2015) Determination of instantaneous pressure in a transonic base flow using four-pulse tomographic PIV. 11th Int Symp Part Image Velocim-PIV15

Charonko JJ, King CV, Smith BL, Vlachos PP (2010) Assessment of pressure field calculations from particle image velocimetry measurements. Meas Sci Technol 21:105401. doi:10.1088/0957-0233/21/10/105401

de Kat R, Ganapathisubramani B (2013) Pressure from particle image velocimetry for convective flows: a Taylor's hypothesis approach. Meas Sci Technol 24:024002. doi: 10.1088/0957-0233/24/2/024002

de Kat R, van Oudheusden BW (2012) Instantaneous planar pressure determination from PIV in turbulent flow. Exp Fluids 52:1089-1106. doi:10.1007/s00348-011-1237-5

Deck S (2005) Zonal-detached-eddy simulation of the flow around a high-lift configuration. AIAA J 43:2372-2384. doi: $10.2514 / 1.16810$

Deck S (2012) Recent improvements in the zonal detached eddy simulation (ZDES) formulation. Theor Comput Fluid Dyn 26:523-550. doi:10.1007/s00162-011-0240-Z

Deck S, Thorigny P (2007) Unsteadiness of an axisymmetric separating-reattaching flow: numerical investigation. Phys Fluids 19:065103. doi:10.1063/1.2734996 
Discetti S, Astarita T (2012) Fast 3D PIV with direct sparse cross-correlations. Exp Fluids 53:1437-1451. doi:10.1007/ s00348-012-1370-9

Discetti S, Natale A, Astarita T (2013) Spatial filtering improved tomographic PIV. Exp Fluids 54: 1505. doi:10.1007/ s00348-013-1505-7

Elsinga GE, Scarano F, Wieneke B, van Oudheusden BW (2006) Tomographic particle image velocimetry. Exp Fluids 41:933947. doi:10.1007/s00348-006-0212-z

Foucaut JM, Carlier J, Stanislas M (2004) PIV optimization for the study of turbulent flow using spectral analysis. Meas Sci Technol 15:1046-1058. doi:10.1088/0957-0233/15/6/003

Gesemann S, Huhn F, Schanz D, Schröder A (2016) From noisy particle tracks to velocity, acceleration and pressure fields using B-splines and penalties, 18th Lisbon Int Symp

Gurka R, Liberzon A, Rubinstein D, Shavit U (1999) Computation of pressure distribution using PIV velocity data. 3rd Int Work Part Image Velocim. Santa Barbara, pp 671-676

Hartley R and Zimmermann A (2003) Multiple view geometry in computer vision, 2nd edn. Cambridge University Press. ISBN: 05215400518

Huhn F, Schanz D, Gesemann S, Schröder A (2016) FFT integration of instantaneous 3D pressure gradient fields measured by Lagrangian particle tracking in turbulent flows. Exp Fluids 57:151. doi:10.1007/s00348-016-2236-3

Jeon YJ, Chatellier L, David L (2014) Fluid trajectory evaluation based on an ensemble-averaged cross-correlation in time-resolved PIV. Exp Fluids 55:1766. doi:10.1007/ s00348-014-1766-9

Jeon YJ, Chatellier L, Beaudoin A, David L (2015) Least-square reconstruction of instantaneous pressure field around a body based on a directly acquired material acceleration in timeresolved PIV. 11th Int Symp Part Image Velocim-PIV15

Laskari A, de Kat R, Ganapathisubramani B (2016) Full-field pressure from snapshot and time-resolved volumetric PIV. Exp Fluids 57:44. doi:10.1007/s00348-016-2129-5

LaVision (2015) Product-manual for DaVis 8.3, item-number: 1105011-4. LaVision, Göttingen

Lecordier B, Westerweel J (2004) The EUROPIV synthetic image generator (S.I.G.). In: Stanislas M, Westerweel J, Kompenhans J (eds) Particle image velocimetry: recent improvements. Springer, Berlin Heidelberg, pp 145-161. doi:10.1007/978-3-642-18795-7_11

Liu X, Katz J (2006) Instantaneous pressure and material acceleration measurements using a four-exposure PIV system. Exp Fluids 41:227-240. doi:10.1007/s00348-006-0152-7

Liu X, Katz J (2013). Vortex-corner interactions in a cavity shear layer elucidated by time-resolved measurements of the pressure field. J Fluid Mech 728, 417-457. doi:10.1017/jfm.2013.275

Lynch K, Scarano F (2013) A high-order time-accurate interrogation method for time-resolved PIV. Meas Sci Technol 24:035305. doi:10.1088/0957-0233/24/3/035305

Lynch KP, Scarano F (2014a) Material acceleration estimation by four-pulse tomo-PIV. Meas Sci Technol 25:084005. doi:10.1088/0957-0233/25/8/084005

Lynch KP, Scarano F (2014b) Experimental determination of tomographic PIV accuracy by a 12-camera system. Meas Sci Technol 25:084003. doi:10.1088/0957-0233/25/8/084003

Lynch KP, Scarano F (2015) An efficient and accurate approach to MTE-MART for time-resolved tomographic PIV. Exp Fluids 56:1-16. doi:10.1007/s00348-015-1934-6

Malik N, Dracos T, Papantoniou D (1993) Particle tracking velocimetry in three-dimensional flows. Exp Fluids 15-15:279-294. doi:10.1007/BF00223406

Manovski P, Depuru Mohan NK, Geisler R, Novara M, Schanz D, Agocs J, Ahlefeldt T, Spehr C, Schr ö der A (2016) 3D
Lagrangian particle tracking using 4-pulse Shake-The-Box synchronised with microphone measurements on a subsonic jet at Mach 0.9. NIOPLEX International Workshop on NonIntrusive Optical Flow Diagnostics, October 25-26, Delft, The Netherlands

Neeteson NJ, Rival DE (2015) Pressure-field extraction on unstructured flow data using a Voronoi tessellation-based networking algorithm: a proof-of-principle study. Exp Fluids 56:44. doi:10.1007/s00348-015-1911-0

Neeteson NJ, Bhattacharya S, Rival DE, Michaelis D, Schanz D, Schröder A (2016) Pressure-field extraction from Lagrangian flow measurements: first experiences with 4D-PTV data. Exp Fluids 57:102. doi:10.1007/s00348-016-2170-4

Novara M, Scarano F (2013) A particle-tracking approach for accurate material derivative measurements with tomographic PIV. Exp Fluids 54:1584. doi:10.1007/s00348-013-1584-5

Novara M, Batenburg KJ, Scarano F (2010) Motion trackingenhanced MART for tomographic PIV. Meas Sci Technol 21:35401. doi:10.1088/0957-0233/21/3/035401

Novara M, Schanz D, Reuther N, Kähler CJ, Schröder A (2016a) Lagrangian 3D particle tracking in high-speed flows: ShakeThe-Box for multi-pulse systems. Exp Fluids 57:128. doi:10.1007/s00348-016-2216-7

Novara M, Schanz D, Gesemann S, Lynch KP, Schröder A (2016b) Lagrangian 3D particle tracking for multi-pulse systems: performance assessment and application of Shake-The-Box, 18th International Symposium on Applications of Laser Techniques to Fluid Mechanics, 4-7 July 2016, Lisbon, Portugal

Ragni D, Schrijer F, van Oudheusden BW, Scarano F (2010) Particle tracer response across shocks measured by PIV. Exp Fluids 50:53-64. doi:10.1007/s00348-010-0892-2

Scarano F (2013) Tomographic PIV: principles and practice. Meas Sci Technol 24:012001. doi:10.1088/0957-0233/24/1/012001

Scarano F, Riethmuller ML (2000) Advances in iterative multigrid PIV image processing. Exp. Fluids 29(7), S51-S60. doi:10.1007/s003480070007

Schanz D, Gesemann S, Schröder A (2016) Shake-The-Box: Lagrangian particle tracking at high particle image densities. Exp Fluids 57:70. doi:10.1007/s00348-016-2157-1

Schneiders JFG, Scarano F (2016) Dense velocity reconstruction from tomographic PTV with material derivatives. Exp Fluids 57:139. doi:10.1007/s00348-016-2225-6

Schneiders JFG, Pröbsting S, Dwight RP, van Oudheusden BW, Scarano F (2016) Pressure estimation from single-snapshot tomographic PIV in a turbulent boundary layer. Exp Fluids 57(4):53. doi:10.1007/s00348-016-2133-9

Schrijer FFJ, Scarano F (2008) Effect of predictor-corrector filtering on the stability and spatial resolution of iterative PIV interrogation. Exp Fluids 45:927-941. doi:10.1007/ s00348-008-0511-7

Schrijer FFJ, Scarano F, van Oudheusden BW (2006) Application of PIV in a Mach 7 double-ramp flow. Exp Fluids 41:353-363. doi:10.1007/s00348-006-0140-y

Schröder A, Geisler R, Staack K, Elsinga G, Scarano F, Wieneke B, Henning A, Poelma C, Westerweel J (2011) Eulerian and Lagrangian views of a turbulent boundary layer flow using time-resolved tomographic PIV. Exp Fluids 50:4. doi:10.1007/ s00348-010-1014-x

Schröder A, Schanz D, Geisler R, Willert C, Michaelis D (2013) Dual-volume and four-pulse Tomo-PIV using polarized light, 10th Symp PIV, Delft, The Netherlands

Souverein LJ, van Oudheusden BW, Scarano F, Dupont P (2009) Application of a dual-plane particle image velocimetry (dualPIV) technique for the unsteadiness characterization of a shock wave turbulent boundary layer interaction. Meas Sci Technol 20:074003. doi:10.1088/0957-0233/20/7/074003 
Theunissen R (2012) Theoretical analysis of direct and phasefiltered cross-correlation response to a sinusoidal displacement for PIV image processing. Meas Sci Technol 23:65302. doi:10.1088/0957-0233/23/6/065302

Tronchin T, David L, Farcy A (2015) Evaluation of pressure field and fluid forces for 3D flow around flapping wing. Exp Fluids 56:1. doi:10.1007/s00348-014-1870-x

van Oudheusden BW (2013) PIV-based pressure measurement. Meas Sci Technol 24:032001. doi:10.1088/0957-0233/24/3/032001

van Oudheusden BW, Scarano F, Roosenboom EWM, Casimeri EWF, Souverein LJ (2007) Evaluation of integral forces and pressure fields from planar velocimetry data for incompressible and compressible flows. Exp Fluids 43:153-162. doi:10.1007/ s00348-007-0261-y
Weiss P-E, Deck S (2011) Control of the antisymmetric mode $(m=1)$ for high Reynolds axisymmetric turbulent separating/reattaching flows. Phys Fluids 23:095102. doi:10.1063/1.3614481

Weiss P-E, Deck S, Robinet J-C, Sagaut P (2009) On the dynamics of axisymmetric turbulent separating/reattaching flows. Phys Fluids 21:075103. doi:10.1063/1.3177352

Westerweel J, Scarano F (2005) Universal outlier detection for PIV data. Exp Fluids 39:1096-1100. doi:10.1007/s00348-005-0016-6

Wieneke B (2008) Volume self-calibration for 3D particle image velocimetry. Exp Fluids 45:549-556. doi:10.1007/ s00348-008-0521-5

Wieneke B (2013) Iterative reconstruction of volumetric particle distribution. Meas Sci Technol 24:024008. doi:10.1088/0957-0233/24/2/024008 